\title{
Eukaryotic elongation factor 2 controls TNF- $\alpha$ translation in LPS-induced hepatitis
}

\author{
Bárbara González-Terán,, José R. Cortés, ${ }^{1}$ Elisa Manieri, ${ }^{1,2}$ Nuria Matesanz, ${ }^{1}$ Ángeles Verdugo, ${ }^{1,2}$
} María E. Rodríguez, ${ }^{1}$ Águeda González-Rodríguez,,3,4 Ángela Valverde,,3,4 Pilar Martín, ${ }^{1}$

Roger J. Davis, ${ }^{5}$ and Guadalupe Sabio'

\begin{abstract}
1Department of Vascular Biology and Inflammation, Fundación Centro Nacional de Investigaciones Cardiovasculares Carlos III, Madrid, Spain. 2Department of Immunology and Oncology, Centro Nacional de Biotecnología/CSIC, Campus de Cantoblanco, Madrid, Spain.

${ }^{3}$ Institute of Biomedicine Alberto Sols (CSIC/UAM), Madrid, Spain. ${ }^{4}$ Centro de Investigación Biomédica en Red de Diabetes y Enfermedades Metabólicas Asociadas, CIBERDEM, ISCIII, Spain. ${ }^{5}$ Howard Hughes Medical Institute and Program in Molecular Medicine, University of Massachusetts Medical School, Worcester, Massachusetts, USA.
\end{abstract}

\begin{abstract}
Bacterial LPS (endotoxin) has been implicated in the pathogenesis of acute liver disease through its induction of the proinflammatory cytokine TNF- $\alpha$. TNF- $\alpha$ is a key determinant of the outcome in a well-established mouse model of acute liver failure during septic shock. One possible mechanism for regulating TNF- $\alpha$ expression is through the control of protein elongation during translation, which would allow rapid cell adaptation to physiological changes. However, the regulation of translational elongation is poorly understood. We found that expression of $\mathrm{p} 38 \gamma / \delta$ MAPK proteins is required for the elongation of nascent TNF- $\alpha$ protein in macrophages. The MKK3/6-p38 $\gamma / \delta$ pathway mediated an inhibitory phosphorylation of eukaryotic elongation factor 2 (eEF2) kinase, which in turn promoted eEF2 activation (dephosphorylation) and subsequent TNF- $\alpha$ elongation. These results identify a new signaling pathway that regulates TNF- $\alpha$ production in LPS-induced liver damage and suggest potential cell-specific therapeutic targets for liver diseases in which TNF- $\alpha$ production is involved.
\end{abstract}

\section{Introduction}

Infection and tissue injury trigger a protective inflammatory response that mobilizes a variety of effector mechanisms to contain and eliminate the injurious agent (1). Hepatic and circulating inflammatory cytokines initiate a positive feedback loop on the innate immune system (2); although cytokine production protects against pathogenic stimuli, it can cause more damage than the initiating event if its magnitude and duration are not strictly controlled by intrinsic negative regulators (1).

The cytokine TNF- $\alpha$ has been identified as a key regulator of the inflammatory response in animal models of fulminant hepatitis (3). Elevated concentrations of this proinflammatory cytokine are also found in acute and chronic inflammatory conditions, including trauma, sepsis, and rheumatoid arthritis (4). Upon binding to its receptors, TNF- $\alpha$ initiates several intracellular signaling cascades that influence cell survival, death, differentiation, proliferation, and migration $(5,6)$.

TNF- $\alpha$ signaling is particularly important in the liver. While it mediates hepatocyte survival and proliferation, it is also implicated in liver failure, since it triggers hepatocyte apoptosis and leads to upregulation of key adhesion molecules and chemokines involved in leukocyte migration and infiltration $(6,7)$. A wellestablished mouse model of acute liver failure and septic shock is the in vivo administration of bacterial LPS and D-galactosamine (D-gal), which strongly induce endogenous TNF- $\alpha$ and other cytokines that cause liver damage $(8,9)$.

TNF- $\alpha$ signaling can be mediated by protein kinases and phosphatases, in particular the MAPK family members ERK, JNK, and p38 MAPK. The p38 MAPK pathway transduces a variety of extracellular signals regulating cellular responses to stress, being implicated in cell proliferation, differentiation, and apop-

Conflict of interest: The authors have declared that no conflict of interest exists. Citation for this article: J Clin Invest. 2013;123(1):164-178. doi:10.1172/JCI65124. tosis $(10,11)$. Four p38 MAPK family members have been identified: $\mathrm{p} 38 \alpha, \mathrm{p} 38 \beta, \mathrm{p} 38 \gamma$, and $\mathrm{p} 38 \delta$, also known as stress-activated kinase 2 a (SAPK2a), SAPK2b, SAPK3, and SAPK4 $(10,12,13)$. The p38 MAPK family can be further divided into 2 subsets, with $\mathrm{p} 38 \alpha$ and $\mathrm{p} 38 \beta$ in one group and p38 $\gamma$ and p38 in the other. All these kinases are activated by double phosphorylation mediated by the upstream kinases MKK3 and MKK6 $(14,15)$. To date, the best-characterized p38 isoform is $\mathrm{p} 38 \alpha$. This isoform is required for macrophage inflammatory responses (1), but in hepatocytes prevents endotoxin-induced liver damage $(16,17)$. Studies using inhibitors suggested that $p 38 \alpha$ and $p 38 \beta$ cooperate in inflammatory processes; however, p38 $\beta$-knockout mice show no differences in several in vivo and in vitro models of inflammation (18). Less is known about the role of the p38 $\gamma$ and p38 MAPK. p38 is known to phosphorylate eukaryotic elongation factor 2 (eEF2) kinase (eEF2K), inhibiting its activity (19). eEF2K blocks mRNA translation into protein through an inhibitory phosphorylation of eEF2.

The rate-limiting step in mRNA translation is thought to be the initiation step; however, far less is known about the regulation of the elongation step of protein biosynthesis (20). The process of peptide-chain elongation is at least partially regulated by eEF2 phosphorylation/dephosphorylation events induced by diverse stimuli (21). Moreover, the phosphorylation state of the upstream kinase eEF2K can be modulated by signaling molecules that regulate the activity of this factor, such as mTOR and MAPKs, including p38 kinases $(19,21-23)$. Because these kinases are activated by different stimuli, it is expected that the regulation of eEF2 will be important in cell adaptation to different physiological states. Here, we show that $\mathrm{p} 38 \delta$ and p38 $\gamma$ expressed in myeloid cells promote eEF 2 activity and, in consequence, TNF- $\alpha$ production and the inflammatory response. By blocking $\mathrm{p} 38 \gamma / \delta$ activity in myeloid macrophages, we were able to suppress hepatitis caused by LPS-induced TNF- $\alpha$ expression. 


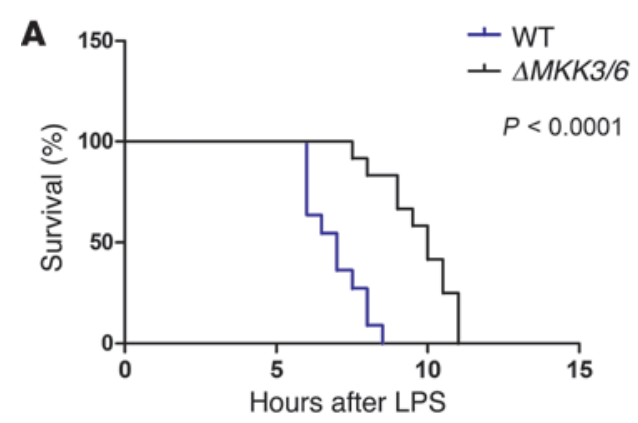

B
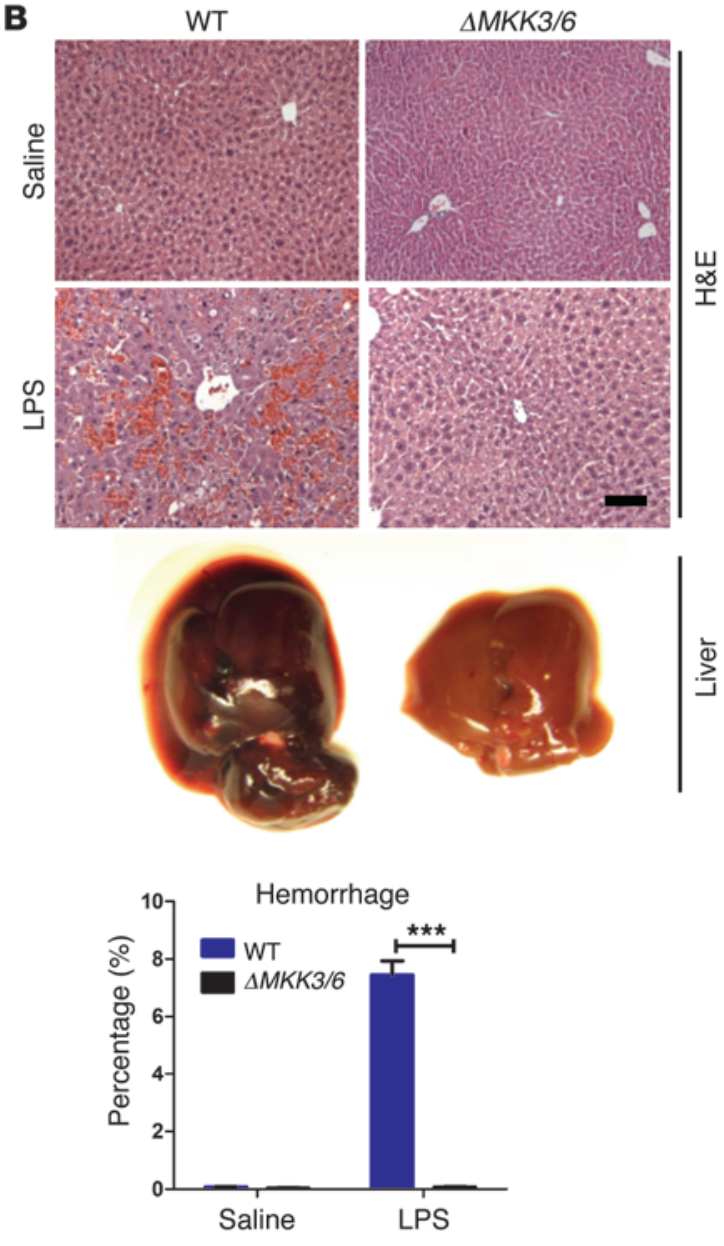

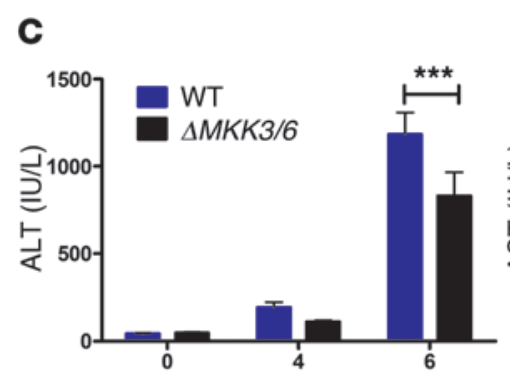

Hours after LPS

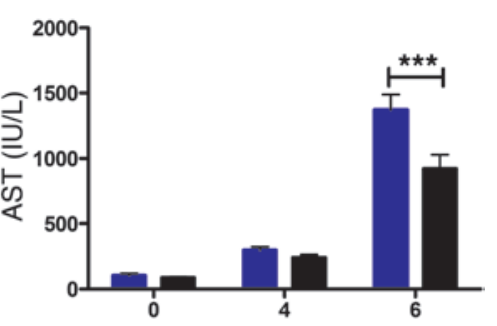

Hours after LPS

D
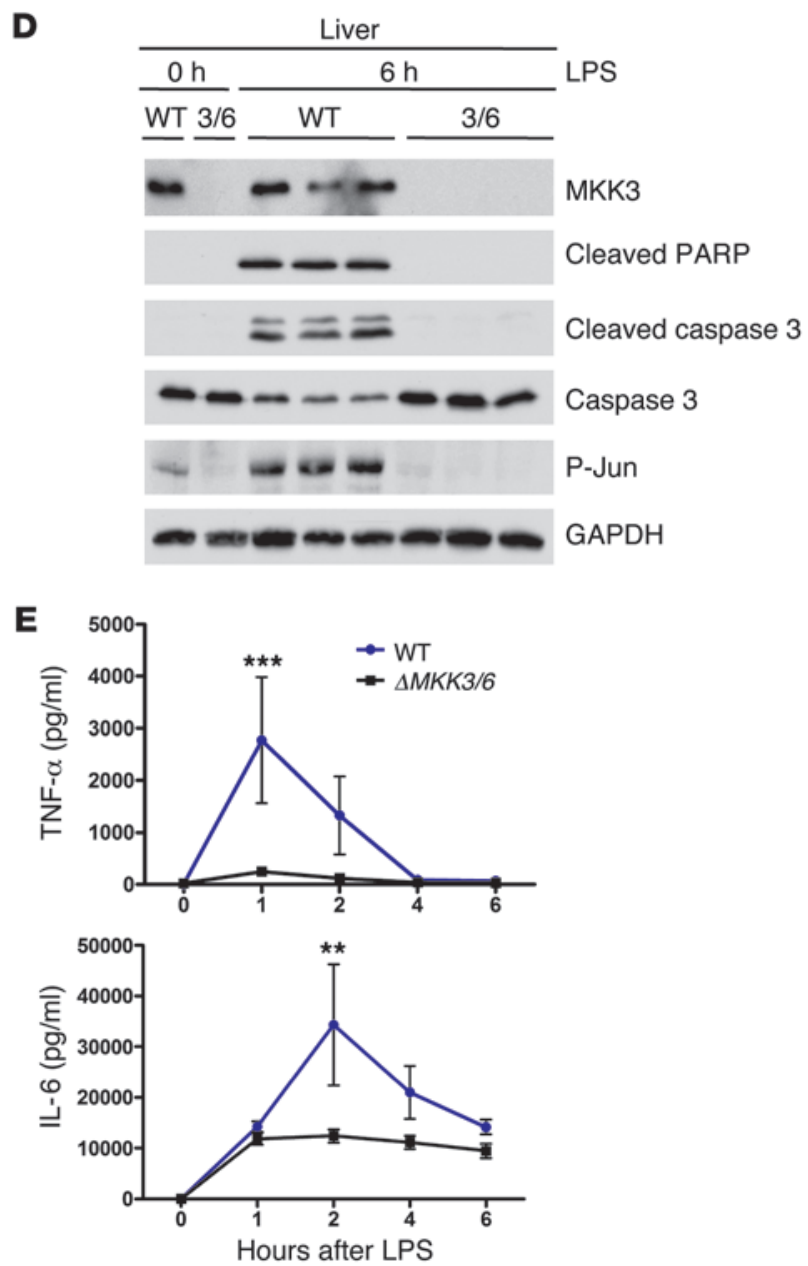

\section{Figure 1}

$\triangle M K K 3 / 6$ mice are protected against LPS-induced liver damage. WT and $M k k 3^{-1-} M k k 6^{-/+}$mice $(\triangle M K K 3 / 6)$ were treated with D-gal+LPS or saline. (A) Survival curves after D-gal+LPS injection $(n=21)$. Survival curves were created with the Kaplan-Meier method and compared by log-rank (MantelCox) test. (B) Livers were removed at 6 hours after injection. Panels show representative H\&E-stained liver sections and livers. The chart presents hemorrhagic area as a percentage of the total area $(n=6-8)$. Scale bar: $50 \mu \mathrm{m}$. (C) Serum transaminase activity at 4 and 6 hours after injection $(n=10)$. (D) Liver extracts were examined by immunoblot with antibodies to cleaved PARP, cleaved caspase 3, caspase 3, phospho-Jun and GAPDH $(n=6-8)$. (E) ELISA analysis of serum TNF- $\alpha$ and IL-6 at different times after injection $(n=16)$. Data are means \pm SD. ${ }^{* *} P<0.01 ;{ }^{* * \star} P<0.001(2-$ way ANOVA coupled to Bonferroni's post tests).

\section{Results}

MKK3 and MKK6 collaborate in LPS-induced hepatitis. To study the role of 38 MAPK signaling in hepatitis, we examined the effect of D-gal+LPS treatment on mice deficient for the upstream kinases MKK3 and MKK6. No differences in mortality where detected in
$\mathrm{Mkk3}^{-{ }^{--}}$or $\mathrm{Mkk6}^{-/-}$mice compared with WT animals (Supplemental Figure 1A; supplemental material available online with this article; doi:10.1172/JCI65124DS1). However, $\mathrm{Mkk3}^{-/-}$mice present less severe liver hemorrhage (Supplemental Figure 1B) and $M k k 6^{-/-}$liver apoptosis was significantly reduced (Supplemental 
Figure 1C). Levels of alanine transaminase (ALT) and aspartate aminotrasferase (AST), 2 well-established markers of hepatic necrosis, were not altered in $M k k 3^{-/-}$mice, but were significantly reduced in $M k k 6^{-1-}$ mice, thus showing an effect on liver necrosis (Supplemental Figure 1D).

The p38 MAPK pathway is a key regulator of the expression of inflammatory cytokines, including IL- 6 and TNF- $\alpha$, the main determinant of LPS-stimulated liver damage (24-27). Circulating levels of TNF- $\alpha$ and IL- 6 after D-gal+LPS injection did not differ between WT mice and the $M k k 3^{-/-}$or $M k k 6^{-1-}$ mice (Supplemental Figure 2A). In vitro stimulation of isolated BM macrophages from these mice with LPS also revealed no differences in proliferation rate or released TNF- $\alpha$ and IL- 6 levels (Supplemental Figure 2, B and C). Moreover, supernatants from WT, $M k k 3^{-/-}$, and $M k k 6^{-/-}$macrophages induce apoptosis similarly in cultured hepatocytes (not shown), thus indicating that the partial protection against LPS-induced liver apoptosis was not due to circulating macrophages.

Neutrophil infiltration can cause mild to severe liver damage (28), and p38 $\alpha$ was recently shown to control neutrophil cytokine production (29). We examined liver myeloid leukocytes based on CD11b, Gr-1, F4/80, and Ly6C markers (Supplemental Figure 2D and ref. 30). Analysis of liver cell populations revealed that D-gal+LPS injection increased liver infiltration by neutrophils $\left(\mathrm{CD} 11 \mathrm{~b}^{+} \mathrm{Gr}-1^{\text {hi }}\right)$ in WT mice, but this effect was impaired in $M k k 3^{---}$and $\mathrm{Mkk6}^{-{ }^{--}}$mice (Supplemental Figure 1E). To investigate the potential involvement of liver neutrophils and macrophages in the protection detected in $M k k 3^{-/-}$and $M k k 6^{-/-}$mice, we isolated leukocytes from the livers of WT, $M k k 3^{--}$, and $\mathrm{Mkk6}^{-/-}$mice and stimulated them with LPS. Whereas the percentage of neutrophils expressing TNF- $\alpha$ was the same in all genotypes, the percentage of monocytes $\left(\mathrm{CD} 11 \mathrm{~b}^{+} \mathrm{Gr}-1^{\text {intermediate }}\right)$ expressing TNF- $\alpha$ was higher in WT mice than in $\mathrm{Mkk3}^{-{ }^{--}}$and $\mathrm{Mkk6}^{-{ }^{--}}$mice (Supplemental Figure $1 \mathrm{~F})$, indicating that the protection against LPS-induced liver apoptosis could be mediated by liver inflammatory monocytes.

$M k k 3^{-1-} M k k 6^{-/+}$mice are protected against LPS-induced liver failure. MKK3 and MKK6 show functional redundancy in the activation of p38 MAPK (14), and while single mutants are viable, doubly deficient $M k k 3^{-/-} M k k 6^{-/-}$and $M k k 3^{-/+} M k k 6^{-/-}$mice die early in embryogenesis. To minimize interference from redundant actions, we used $M k k 3^{-/-} M k k 6^{-{ }^{+}}(\Delta \mathrm{MKK} 3 / 6)$ mice, which are viable (14). As expected, $\triangle \mathrm{MKK} 3 / 6$ mice were protected against LPS-induced hepatotoxicity, showing milder hemorrhage and better survival than WT mice (Figure 1, A and B). Immunoblot analysis demonstrated that D-gal+LPS injection activated caspase 3 and PARP cleavage in WT liver but not in $\triangle \mathrm{MKK} 3 / 6$ livers (Figure $1 \mathrm{D}$ ). Moreover, circulating transaminases were reduced in $\triangle \mathrm{MKK} 3 / 6$ mice, indicating a reduction in liver necrosis (Figure 1C). These data demonstrate that MKK3/6 deficiency severely limits LPSstimulated hepatic apoptosis. Examination of serum TNF- $\alpha$ after $D$-gal+LPS injection revealed that the marked increase in TNF- $\alpha$ concentration observed in WT and singly deficient mice was not detected in $\triangle \mathrm{MKK} 3 / 6$ mice (Figure 1E). Serum IL- 6 was also lower in $\Delta \mathrm{MKK} 3 / 6$ mice (Figure $1 \mathrm{E}$ ). The loss of LPS-stimulated TNF- $\alpha$ expression in $\triangle \mathrm{MKK} 3 / 6$ mice might account for the finding that LPS activation of JNK pathway in the livers of WT mice was not seen in $\triangle \mathrm{MKK} 3 / 6$ mice (Figure 1D).

$M K K 3$ and $M K K 6$ are required for neutrophil migration after LPS injection. The lower TNF- $\alpha$ production in $\triangle \mathrm{MKK} 3 / 6$ mice could be a consequence of the lower leukocyte infiltration in liver. To address this, we analyzed myeloid cell migration after D-gal+LPS injection. Quantification of liver chemokines showed that in WT mice, LPS treatment increased mRNA expression of molecules important for migration of macrophages (MCP1 and ICAM) and neutrophils (KC, MIP2), whereas induction of these chemokines and adhesion molecules was decreased in the livers of $\triangle \mathrm{MKK} 3 / 6$ mice (Figure 2A). Phenotypic characterization of liver-infiltrating leukocytes from control and D-gal+LPS-treated livers revealed a significant increase in neutrophils in the inflammatory cell infiltrate of WT mice that was impaired in $\Delta \mathrm{MKK} 3 / 6$ mice (Figure $2 \mathrm{~B}$ ), consistent with the reduced disease severity (28). However, the percentages of macrophages and monocytes did not differ between genotypes (Figure 2B). These findings correlated with lower RNA expression of neutrophil markers (Elastase 2, Ly6G) in the livers of LPS-injected $\Delta \mathrm{MKK} 3 / 6$ mice (Supplemental Figure $3 \mathrm{~A}$ ).

The reduced liver neutrophil infiltration in $\Delta \mathrm{MKK} 3 / 6$ mice could be due either to impaired neutrophil release from BM or to a defect in cell migration. The levels of circulating neutrophils after LPS injection showed a sharp increase in WT mice that was absent in $\triangle \mathrm{MKK} 3 / 6$ mice (Figure $2 \mathrm{C}$ ). These data show that the reduced neutrophil infiltration in liver is in part caused by lowered neutrophil mobilization from the BM. To investigate whether the low circulating levels of TNF- $\alpha$ in $\Delta \mathrm{MKK} 3 / 6$ mice might be responsible for this alteration, we injected TNF- $\alpha+D$-gal into WT and $\triangle \mathrm{MKK} 3 / 6$ mice, resulting in increased circulating neutrophil levels in both genotypes (Supplemental Figure 3B). These data indicate that the defect in migration is secondary to the low production of TNF- $\alpha$ in $\triangle \mathrm{MKK} 3 / 6$ mice.

$M K K 3$ and MKK 6 regulate TNF- $\alpha$ production by macrophages. To investigate the molecular mechanism underlying reduced circulating TNF- $\alpha$ in $\Delta \mathrm{MKK} 3 / 6$ mice, we treated BM-derived macrophages from WT and $\triangle \mathrm{MKK} 3 / 6$ mice with LPS. Whereas treatment of WT macrophages with LPS activated JNK, ERK, and p38 MAPK, in $\triangle \mathrm{MKK} 3 / 6$ macrophages the activation of p38 MAPK was impaired (Supplemental Figure $3 \mathrm{C}$ ). The $\Delta \mathrm{MKK} 3 / 6$ macrophages also secreted markedly reduced amounts of TNF- $\alpha$ and IL-6 compared with WT macrophages, confirming that MKK3/6 deficiency causes major defects in TNF- $\alpha$ production (Supplemental Figure 3D). Further analysis showed that neutrophils and Kupffer cells, 2 other myeloid cell types implicated in liver damage, also secreted reduced amounts of TNF- $\alpha$ and IL-6 (Supplemental Figure 3, E and F). However, in vivo analysis indicated that although the percentage of liver neutrophils expressing TNF- $\alpha$ after D-gal+LPS injection was the same in both genotypes, the percentage of monocytes and macrophages expressing TNF- $\alpha$ was higher in WT livers (Figure 2D).

To confirm that reduced cytokine production by liver macrophages is responsible for the protection against hepatocyte apoptosis in $\Delta \mathrm{MKK} 3 / 6$ mice, we stimulated primary hepatocytes with conditioned medium from LPS-treated BM-derived macrophages. Conditioned medium from LPS-treated WT macrophages increased hepatocyte apoptosis, measured by cleavage of caspase 3 and PARP, whereas conditioned medium from LPS-treated $\triangle \mathrm{MKK} 3 / 6$ macrophages had no effect (Supplemental Figure 3G).

$M K K 3$ and $M K K 6$ are not required for TNF- $\alpha-$ induced liver damage. Our results so far show that MKK3/6 deficiency causes decreased TNF- $\alpha$ production in a TNF- $\alpha$-dependent hepatitis model. This implies that $\Delta \mathrm{MKK} 3 / 6$ mice are defective for TNF- $\alpha$ secretion, but not TNF- $\alpha$ responsiveness. To test this, we treated mice with TNF- $\alpha$, finding that TNF- $\alpha$ caused similar mortality and liver damage in 
A

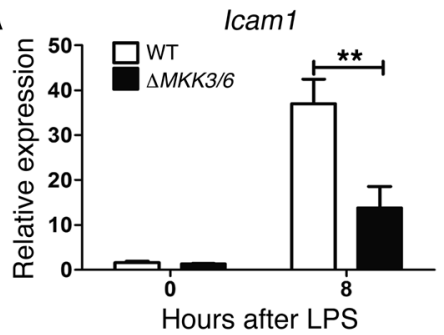

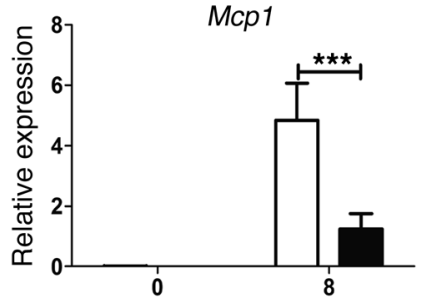

Hours after LPS

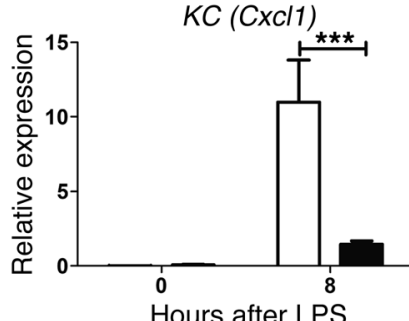

Hours after LPS
Mip2 (Cxcl2)

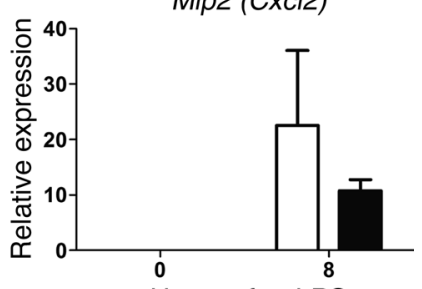

Hours after LPS
B

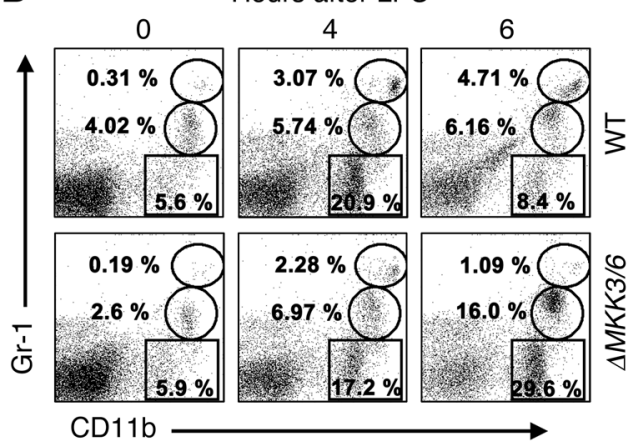

C

Circulating neutrophils

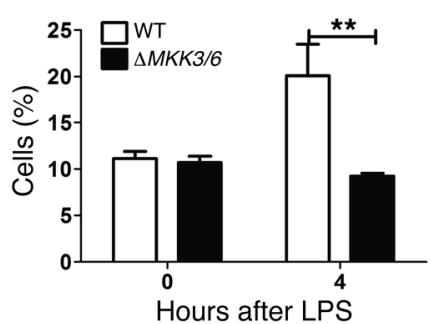

Figure 2

$\triangle M K K 3 / 6$ mice have lower liver inflammation after LPS injection. WT and $\triangle M K K 3 / 6$ mice were treated with D-gal+LPS or saline. (A) Total RNA was extracted from livers 6 hours after treatment and chemokine mRNA levels determined by qRT-PCR. mRNA expression was normalized to Gapdh ( $n=5-8)$. (B) Liver myeloid subsets $(C D 11 b+G r-1$ hi, CD11b+Gr-1 intermediate, $\left.\mathrm{CD} 11 \mathrm{~b}^{+} \mathrm{Gr}-1^{-}\right)$were assessed by flow cytometry of liver leukocytes isolated from WT and $\triangle M K K 3 / 6$ mice 4 and 6 hours after treatment. Representative dot plots are shown. Bar charts show each myeloid population as the percentage of total intrahepatic leukocyte population $(n=7)$. (C) Neutrophils as a percentage of circulating leukocytes, measured in total blood 4 hours after injection ( $n=5-8)$. (D) TNF- $\alpha$ and IL-12 production by liver myeloid subsets were analyzed by intracellular staining in neutrophils $\left(C D 11 b^{+}\right.$ Gr-1 $\left.{ }^{\text {hi }}\right)$, monocytes (CD11b+Gr-1 intermediate) and CD11 $b^{+} \mathrm{Gr}-1-$ myeloid cells isolated from WT and $\triangle M K K 3 / 6$ mice 4 and 6 hours after injection. Representative dot plots are shown for all treatment groups, and bar charts show TNF- $\alpha$-positive cells as the percentage of each myeloid population $(n=7)$. Data are means $\pm \mathrm{SD} .{ }^{*} P<0.05$; ${ }^{\star \star} P<0.01 ;{ }^{* \star} P<0.001$ (2-way ANOVA coupled to Bonferroni's post tests).

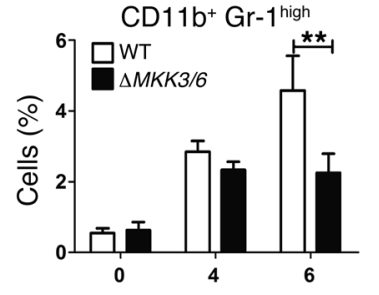

Hours after LPS

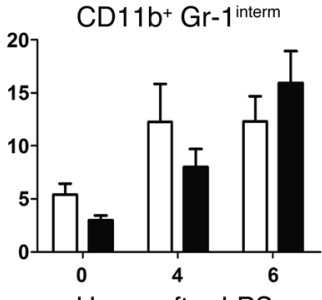

Hours after LPS

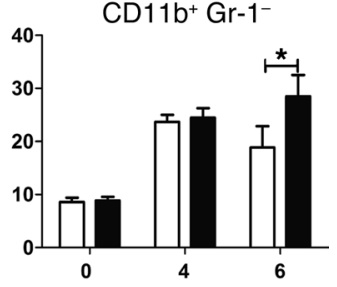

Hours after LPS
Hours after LPS

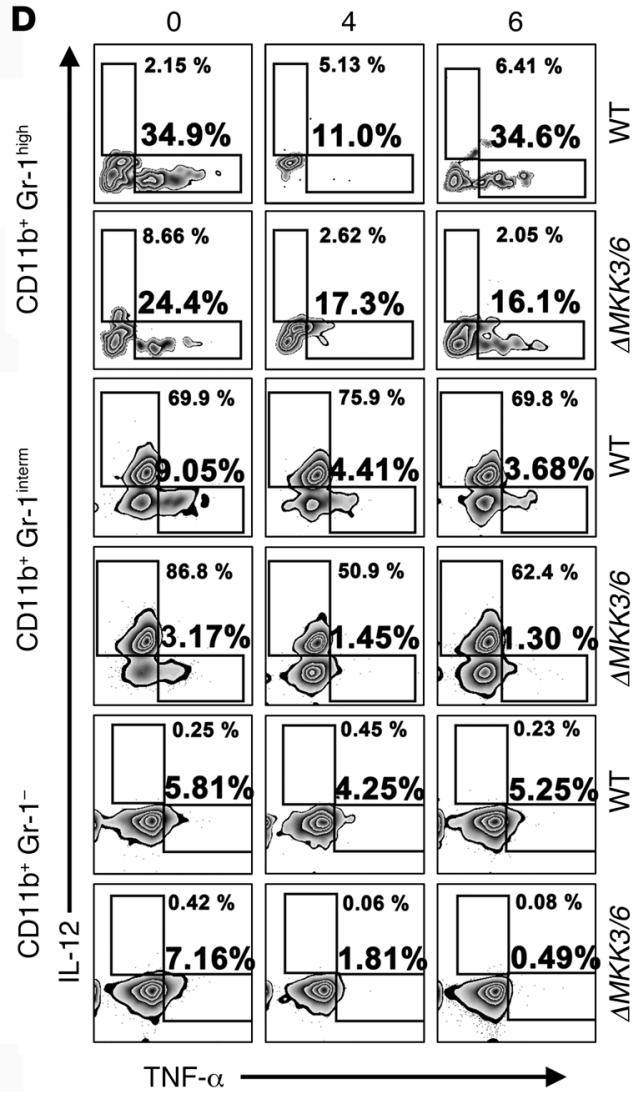

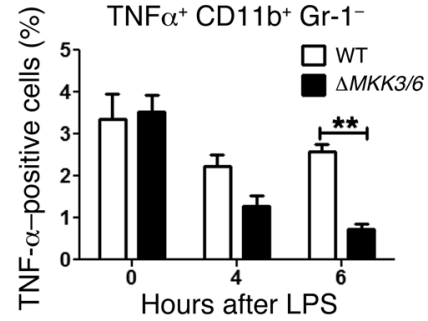
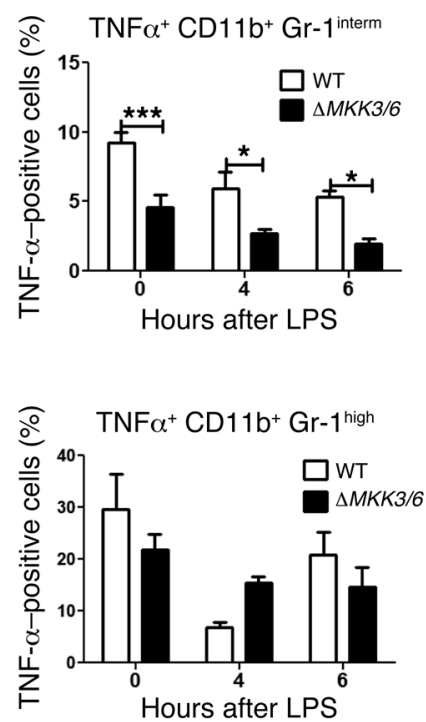
A

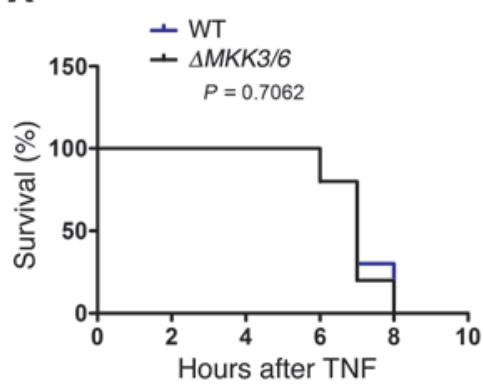

B

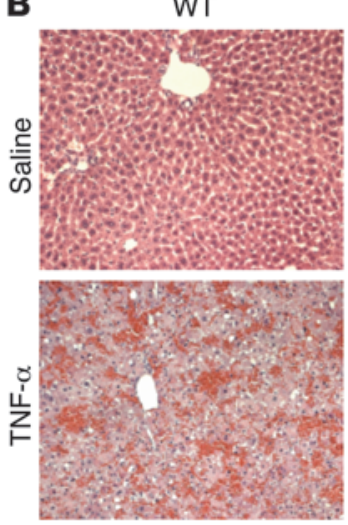

$\Delta M K K 3 / 6$

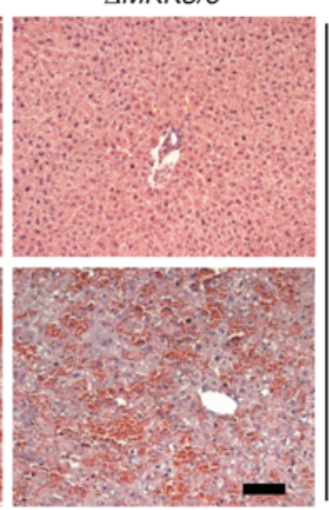

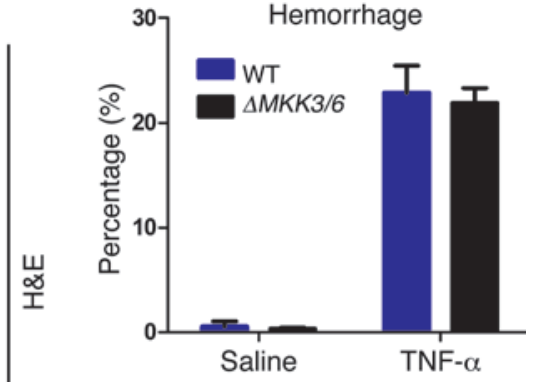

D
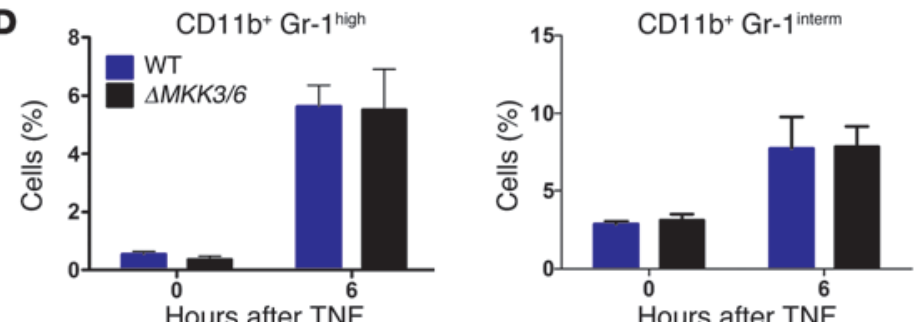

Hours after TNF
C

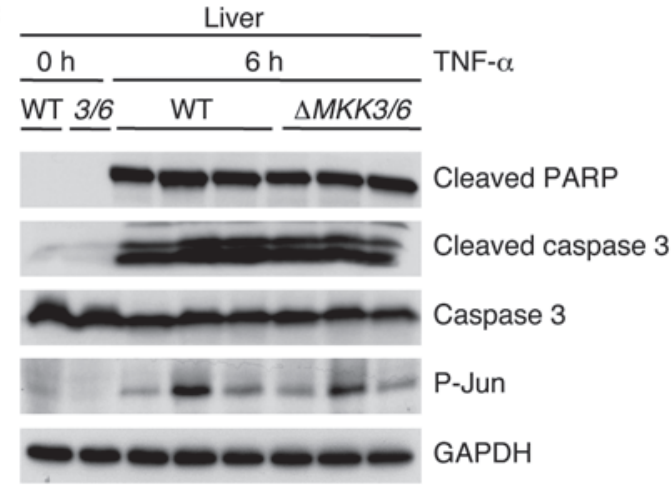

E

\begin{tabular}{|c|c|c|}
\hline \multicolumn{3}{|c|}{ Primary hepatocytes } \\
\hline WT & $\Delta M K K 3 / 6$ & \\
\hline $0 \mathrm{~h} 24 \mathrm{~h}$ & $0 \mathrm{~h} 24 \mathrm{~h}$ & TNF- $\alpha+\mathrm{CHX}$ \\
\hline$-=$ & $=$ & Cleaved caspase 3 \\
\hline-- & -1 & Caspase 3 \\
\hline-- & $-\cdots$ & GAPDH \\
\hline
\end{tabular}
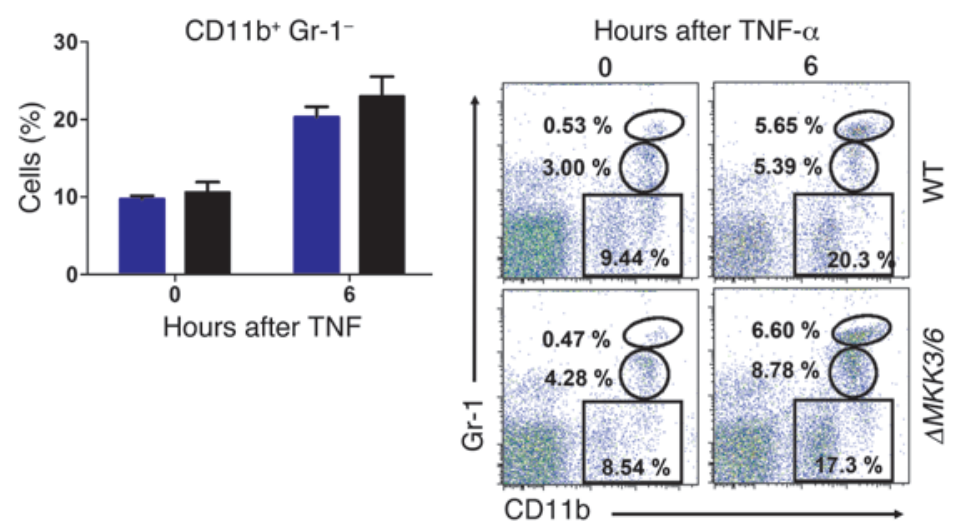

\section{Figure 3}

$\triangle M K K 3 / 6$ mice are not protected against TNF- $\alpha$-induced liver damage. WT and $\Delta M K K 3 / 6$ mice were i.v. injected with $10 \mu \mathrm{g} / \mathrm{kg}$ TNF- $\alpha$ plus $1 \mathrm{~g} / \mathrm{kg} \mathrm{D}-\mathrm{gal}$ (TNF- $\alpha /$ Gal) or with saline. (A) Survival curves after TNF- $\alpha / G a l$ injection $(n=13)$. Survival curves were created with the Kaplan-Meier method and compared by log-rank (Mantel-Cox) test. (B) Representative H\&E-stained sections of livers extracted 6 hours after injection. The chart presents hemorrhagic area as a percentage of the total area $(n=8)$. Scale bar: $50 \mu \mathrm{m}$. (C) Liver extracts were examined by immunoblot analysis for cleaved PARP,

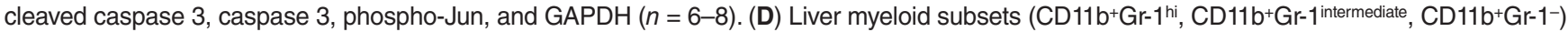
were assessed by flow cytometry of liver leukocytes isolated from WT and $\triangle M K K 3 / 6$ mice 4 and 6 hours after treatment. Representative dot plots are shown. Bar charts show each myeloid subset as a percentage of the total intrahepatic leukocyte population $(n=4-6)$. (E) Immunoblot of cleaved caspase 3, caspase 3, and GAPDH in WT and $\triangle M K K 3 / 6$ primary hepatocytes treated with TNF- $\alpha(20 \mathrm{ng} / \mathrm{ml}) \mathrm{plus} \mathrm{CHX}(100 \mu \mathrm{g} / \mathrm{ml})$. Data are means \pm SD. No significant differences were found (2-way ANOVA coupled to Bonferroni's post tests).

WT and $\triangle \mathrm{MKK} 3 / 6$ mice (Figure $3, \mathrm{~A}$ and $\mathrm{B}$ ). Immunoblot analysis demonstrated that TNF- $\alpha$ stimulated a similar degree of caspase 3 and PARP cleavage (Figure 3C). Moreover, TNF- $\alpha$ induced the same levels of neutrophil liver infiltration in both genotypes (Figure 3D). To confirm that the protection was due to defective cytokine production and not to a protective loss of responsiveness to TNF- $\alpha$, we treated primary hepatocytes from WT or $\Delta \mathrm{MKK} 3 / 6$ mice with TNF- $\alpha$ and the protein synthesis inhibitor cycloheximide (CHX). Both hepatocyte populations presented the same level of apoptosis (Figure $3 \mathrm{E}$ ). These data demonstrate that $\Delta \mathrm{MKK} 3 / 6$ mice do not exhibit resistance to TNF- $\alpha$-induced hepatitis and indicate that the primary protection against LPS-induced hepatitis in these mice is due to an inability to produce TNF- $\alpha$.

$M K K 3$ and $M K K 6$ are required in the bematopoietic compartment for LPS-induced liver damage. The source of TNF- $\alpha$ in mouse models of hepatitis is the hematopoietic compartment $(31,32)$. There- 
A
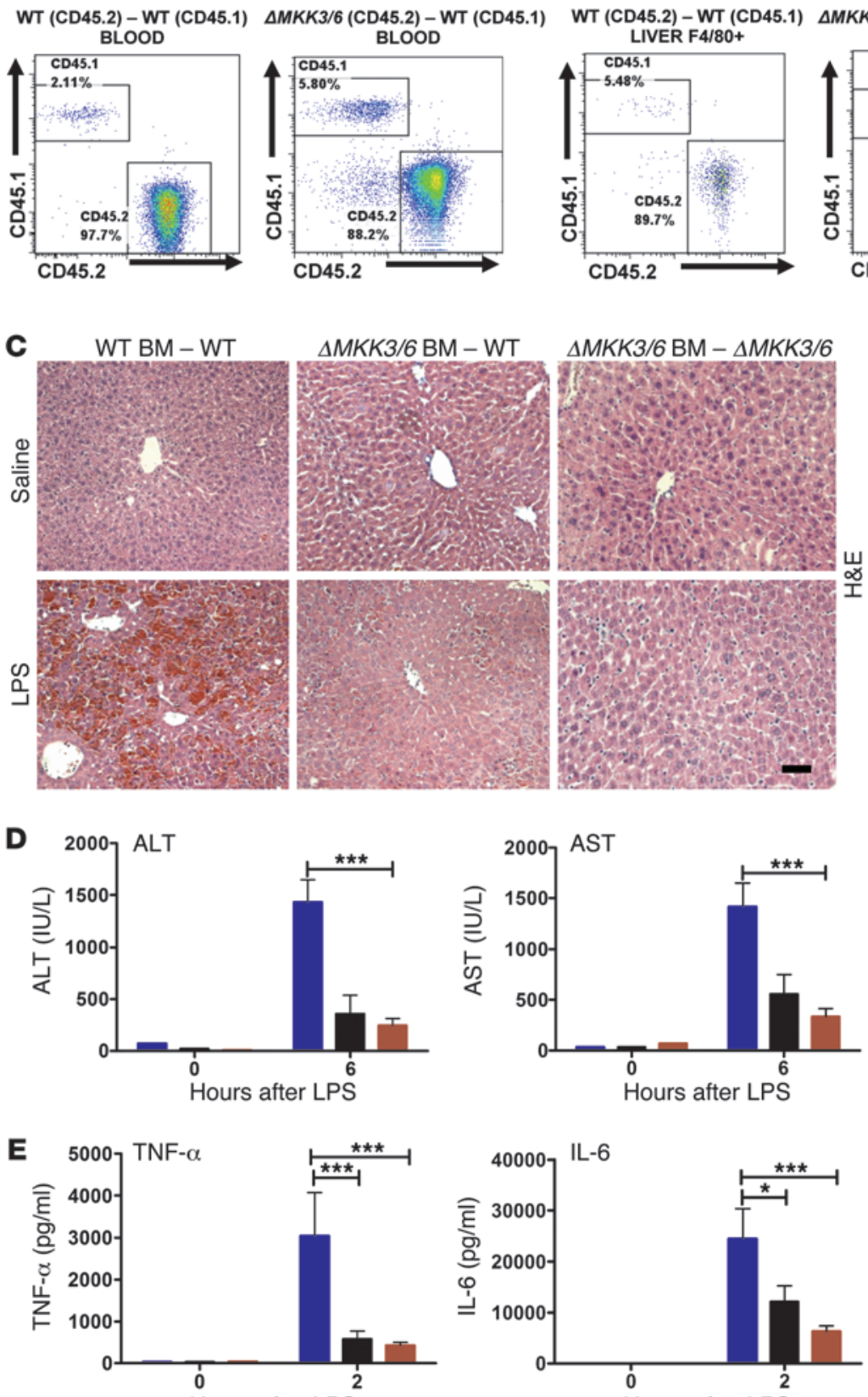

Hours after LPS
B
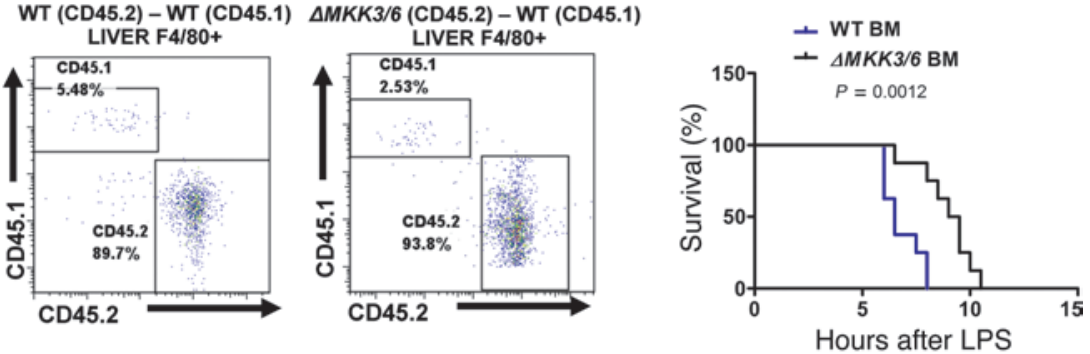

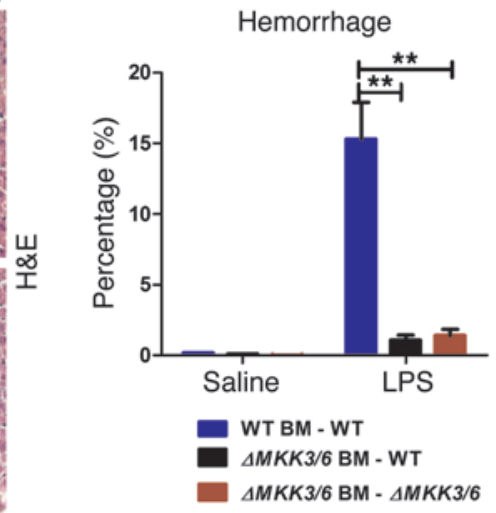

\section{Figure 4}

$\triangle M K K 3 / 6$ hematopoietic cells protect mice against hepatitis. Lethally irradiated WT mice were reconstituted with BM from WT or $\triangle M K K 3 / 6$ mice. Mice were treated with D-gal+LPS 2 months after transplantation. (A) $2 \times 10^{7}$ freshly prepared CD45.2 whole BM mononuclear cells were transplanted into lethally irradiated B6.SJL (CD45.1) mice, and engraftment by CD45.2 cells (\%) was analyzed by antibody staining and FACS of peripheral blood after 4 months. Left panels: representative FACS dot plot of CD45.1 and CD45.2 expression in cells isolated from the blood of transplanted mice. Right panels: representative FACS dot plot of CD45.1 and CD45.2 expression in F4/80-positive cells isolated from the liver of transplanted mice $(n=3)$. (B) Survival curves after D-gal+LPS injection $(n=10)$. Survival curves were created with the Kaplan-Meier method and compared by log-rank (Mantel-Cox) test. (C) Representative H\&E-stained sections of livers extracted 6 hours after injection. The chart presents hemorrhagic area as a percentage of the total area $(n=5-8)$. Scale bar: $50 \mu \mathrm{m}$. (D) Serum transaminase activity at 4 and 6 hours after injection $(n=5-8)$. (E) ELISA of serum TNF- $\alpha$ and IL- 6 at different times after injection $(n=5-8)$. Data are means \pm SD. ${ }^{*} P<0.05$; ${ }^{* *} P<0.01 ;{ }^{* *} P<0.001$ (2-way ANOVA coupled to Bonferroni's post tests). 

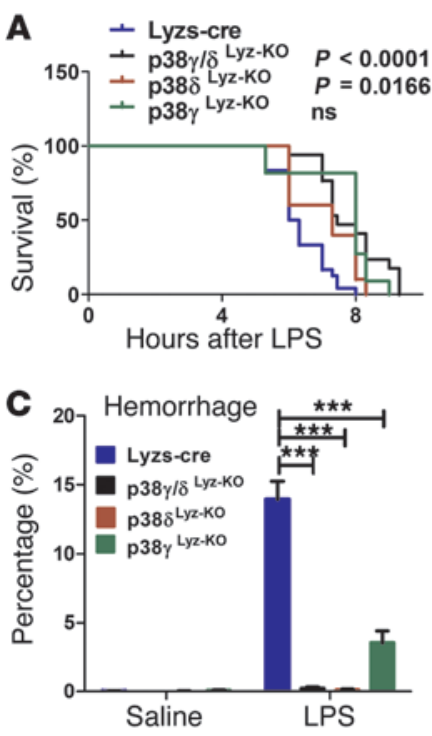

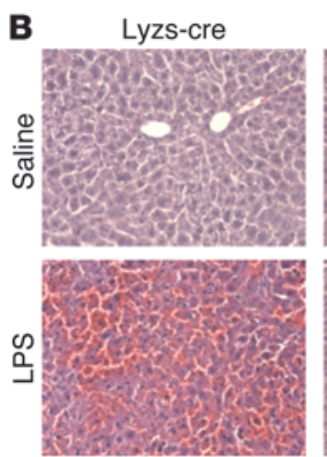

p38 $\gamma / \delta^{\text {Lyz-Ko }}$
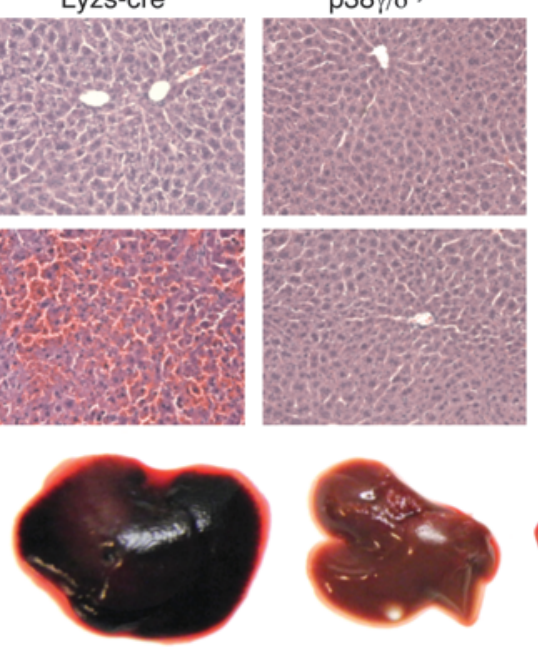

p38 $\delta^{\text {Lyz-KO }}$
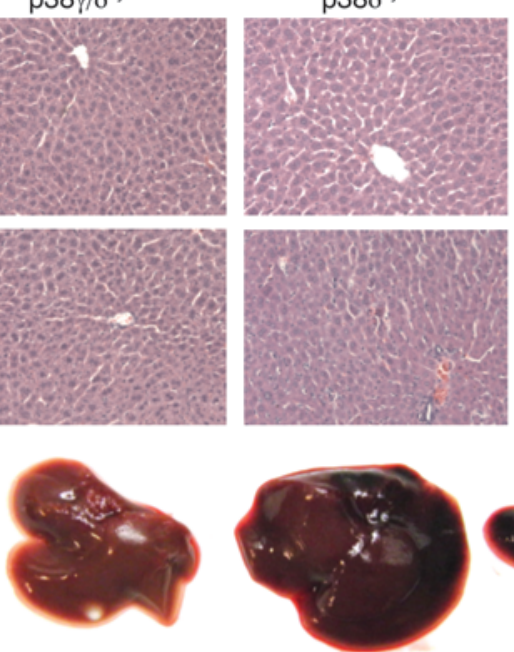

p38 $\gamma^{\text {Lyz-KO }}$

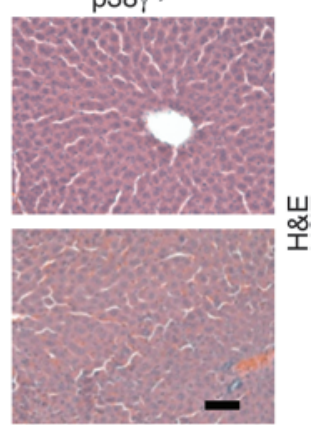

Liver

\begin{tabular}{|c|c|c|c|c|c|c|c|c|c|}
\hline \multicolumn{2}{|r|}{ Lyzs-cre } & \multicolumn{2}{|c|}{$\mathrm{p} 38 \gamma / \delta^{\mathrm{Lyz}-K O}$} & \multicolumn{2}{|r|}{ Lyzs-cre } & p38 $\delta^{\text {Lyz-KO }}$ & Lyzs-cre & p38 $\gamma^{\text {Lyz-KO }}$ & \\
\hline- & + & - & + & - & + & + & + & + & \\
\hline & --- & & & & 는 & $--\div$ & $\cdots-\infty$ & $--\infty$ & Cleaved PARP \\
\hline & $=5-$ & & $=$ & & $-==$ & $=-=$ & $-==$ & $-=-$ & Cleaved caspase 3 \\
\hline - & --1 & - & --- & - & $-2=$ & $-=-2$ & ---- & $---\cdots$ & Caspase 3 \\
\hline & --- & 一 & --- & - & ---1 & ----0 & $-\cdots$ & ---1 & GAPDH \\
\hline
\end{tabular}

E

AST
ALT

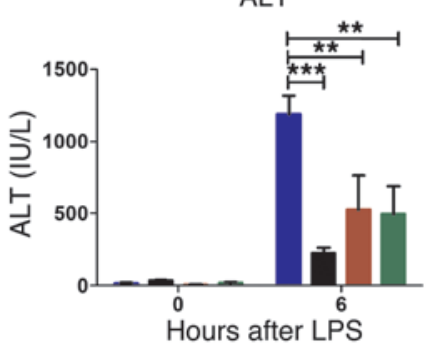

F
IL-6

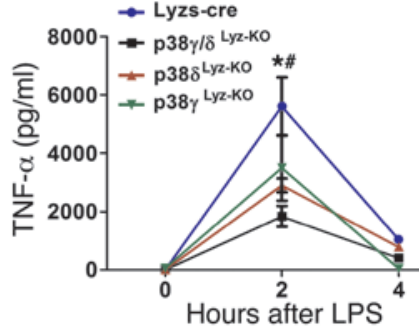

G

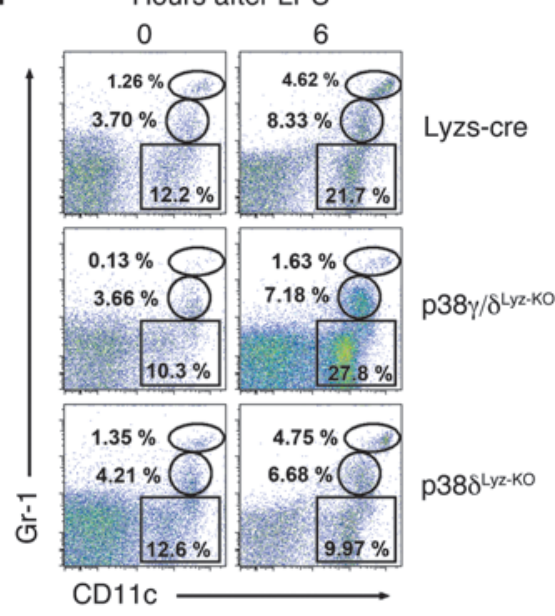

CD11b+Gr-1 high $^{\text {he }}$
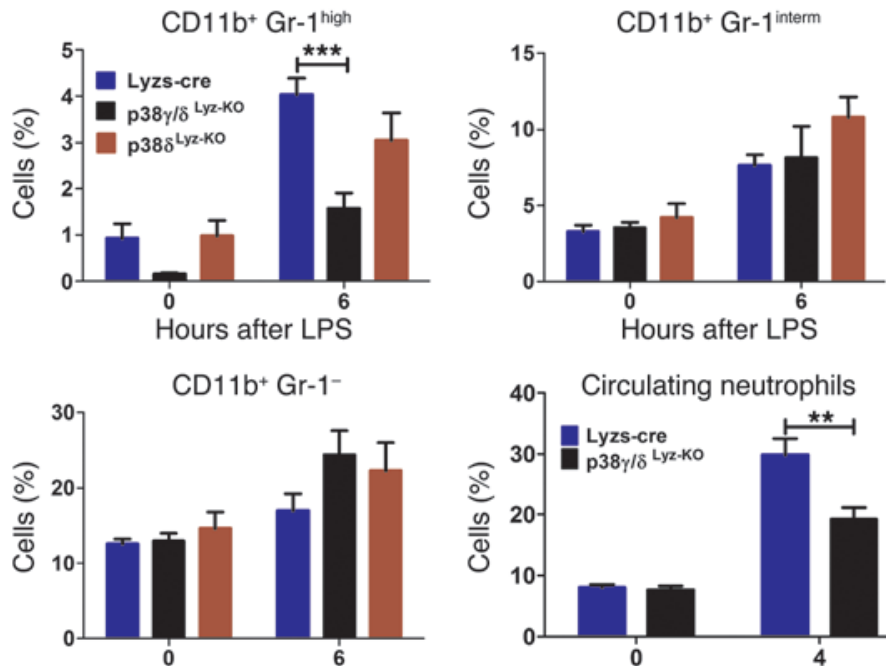

Hours after LPS

Hours after LPS
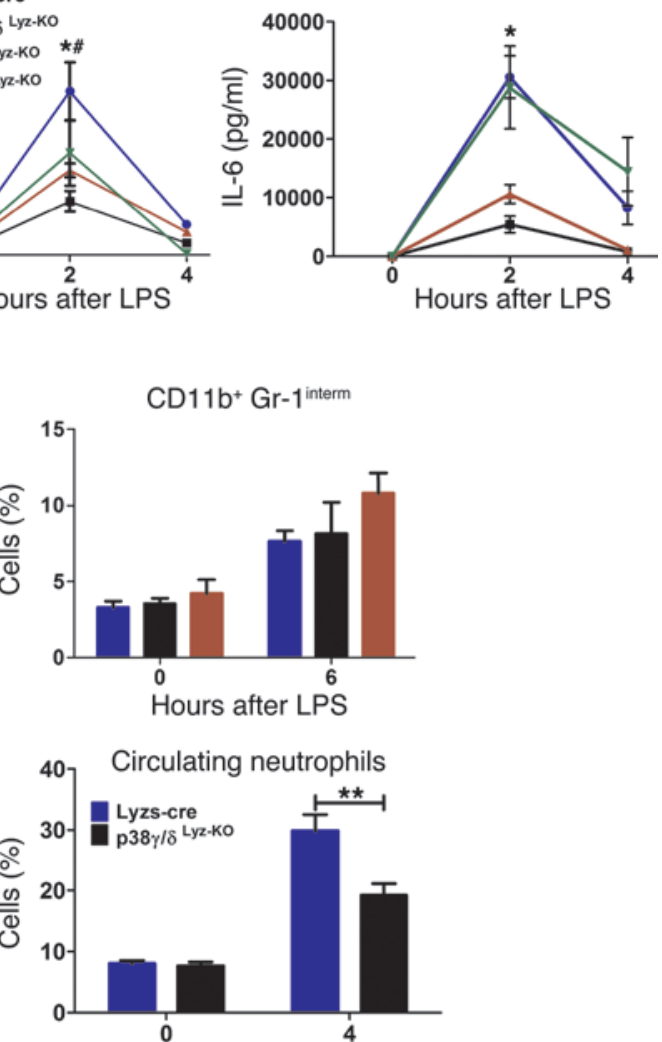


\section{Figure 5}

p38 $\gamma / \delta^{L y z-K O}$ mice are protected against LPS-induced liver damage. p38 $\gamma^{\text {Lyz-KO }}$, p38 $\delta^{\text {Lyz-KO }}$, p38 $\gamma / \delta^{\text {Lyz-KO }}$, and control Lyzs-cre transgenic mice were injected with D-gal+LPS or saline. (A) Mouse survival after D-gal+LPS injection $(n=14)$. Survival curves were created with the Kaplan-Meier method and compared by log-rank (Mantel-Cox) test. (B) Livers were removed at 6 hours after injection. Panels show representative H\&E-stained liver sections and livers $(n=5-8)$. Scale bar: $50 \mu \mathrm{m}$. (C) Hemorrhagic area as a percentage of the total area on H\&E-stained liver sections ( $n=5-8)$. (D) Immunoblot analysis of liver extracts $(n=5-8)$. (E) Serum transaminase activity at 6 hours after injection $(n=8-10)$. $(\mathbf{F})$ ELISA of serum TNF- $\alpha$ and IL- 6 at different times after injection $(n=10)$. ${ }^{\#} P<0.01$ for Lyzs-cre versus p38 $\delta^{\text {Lyz-KO }}$ mice in TNF- $\alpha$; ${ }^{*} P<0.001$ for

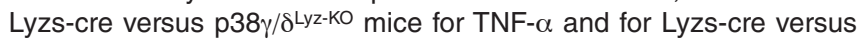
$\mathrm{p} 38 \gamma / \delta^{\mathrm{Lyz}-K O}$ or $\mathrm{p} 38 \delta^{\mathrm{Lyz}-K O}$ mice in IL-6 $(n=6-8)$. (G) Liver myeloid subsets $\left(C D 11 b^{+} G r-1\right.$ hi, $\left.C D 11 b^{+} G r-1^{\text {intermediate }}, C D 11 b^{+} G r-1^{-}\right)$were assessed by flow cytometry of liver leukocytes isolated from p38 $\gamma^{L y z-K O}$, p38 $3 \delta^{\text {Lyz-KO }}$, p38 $\gamma / \delta^{\text {Lyz-KO }}$, and control Lyzs-cre transgenic mice 6 hours after injection. Representative dot plots are shown. Bar charts show each myeloid population as a percentage of the total intrahepatic leukocyte population (mean \pm SD; $n=4-6$ ). Circulating neutrophils in total blood were measured as a percentage of circulating leukocytes 4 hours after injection $(n=5-8)$. Data are means \pm SD. ${ }^{* *} P<0.01 ;{ }^{* *} P<0.001$ (2-way ANOVA coupled to Bonferroni's post tests).

fore, if defective TNF- $\alpha$ expression accounts for the protection of $\Delta \mathrm{MKK} 3 / 6$ mice against TNF- $\alpha$-dependent hepatitis, transplantation of the hematopoietic compartment of $\Delta \mathrm{MKK} 3 / 6$ mice to immunodeficient donors should confer this protection. To test the role of hematopoietic cells, we constructed radiation chimeras by transplanting $\mathrm{BM}$ from WT and $\Delta \mathrm{MKK} 3 / 6$ donor mice into lethally irradiated congenic WT and $\Delta \mathrm{MKK} 3 / 6$ recipients. Efficient reconstitution of B6.SJL (CD45.1) mice with BM from WT C57BL/6J (CD45.2) or $\Delta \mathrm{MKK} 3 / 6$ (CD45.2) mice was confirmed by flow cytometry analysis of peripheral blood leukocytes stained with antibodies to CD45.1/CD45.2. Moreover, analysis of Kupffer cells $\left(\mathrm{F} 4 / 80^{+}\right)$in the liver of recipients after transplantation showed that more than $90 \%$ of Kupffer cells originated from the donor mice (Figure 4A).

We found that lethally irradiated mice reconstituted with $\triangle \mathrm{MKK} 3 / 6 \mathrm{BM}$ were indeed protected against LPS-induced mortality and exhibited lower levels of LPS-stimulated serum TNF- $\alpha$ and hepatic damage than mice reconstituted with WT BM (Figure 4, B-E). These data confirm that MKK3 and MKK6 expressed in hematopoietic cells play a critical role in the expression of TNF- $\alpha$ that leads to the development of hepatitis.

Inbibition of $p 38 \gamma$ and $p 38 \delta$ protects against LPS-induced hepatitis. MKK 3 and MKK 6 are the main regulators of the $\alpha, \beta, \gamma$, and $\delta$ p38 MAPK isoforms. To determine which isoforms mediate MKK3/MKK6-induced TNF- $\alpha$ expression, we analyzed the effect on D-gal+LPS-induced liver damage of 2 inhibitors: SB203580, which inhibits $\mathrm{p} 38 \alpha$ and $\mathrm{p} 38 \beta$, and BIRB796, which inhibits all isoforms (33). We treated mice with BIRB796, SB203580, or DMSO prior to LPS injection. We first examined the specific action of these inhibitors on MAPK pathway activity. Both compounds efficiently inhibited $\mathrm{p} 38 \alpha / \beta$, as shown by the loss of the phosphorylation of the known p $38 \alpha$ substrate HSP27 (Supplemental Figure 4A and ref. 34). In contrast, only BIRB796 was able to inhibit $\mathrm{p} 38 \delta$ activation, as indicated by suppression of the phosphorylation of its substrate, eEF2K (19). No changes in ERK or JNK phosphorylation were observed (Supplemental Figure 4A).
Injection of BIRB796 into WT mice protected against D-gal+LPSinduced liver damage, whereas SB203580 exacerbated liver damage (hemorrhage, apoptosis, and necrosis) (Supplemental Figure 4 , B-D). Injection of BIRB796 also reduced serum levels of IL-6 and TNF- $\alpha$ in D-gal+LPS-treated mice (Supplemental Figure $4 \mathrm{E})$. These results suggest that LPS-induced liver injury might be mediated by $\mathrm{p} 38 \gamma$ and $\mathrm{p} 38 \delta$.

Myeloid expression of $p 38 \gamma$ and $p 38 \delta$ is required for LPS-induced hepatitis. To investigate the role of p38 $\gamma$ and p38 in LPS-induced hepatitis, we generated mice with myeloid cell-specific ablation of these p38 isoforms, separately and in combination. Mice carrying conditional loxP-flanked alleles for p38 $\gamma, \mathrm{p} 38 \delta$, and p38 $\gamma / \delta\left(\mathrm{p} 38 \gamma^{\mathrm{fl}}\right.$, $\mathrm{p} 38 \delta^{\mathrm{fl}}$, and $\mathrm{p} 38 \gamma / \delta^{\mathrm{fl}}$ mice) were crossed with Lyzs-cre transgenic mice (35), yielding $\mathrm{p} 38 \gamma^{\mathrm{Lyz}-\mathrm{KO}}, \mathrm{p} 38 \delta^{\mathrm{Lyz}-\mathrm{KO}}$, and $\mathrm{p} 38 \gamma / \delta^{\mathrm{Lyz}-\mathrm{KO}}$ mice. Immunoblot analysis confirmed efficient ablation of the appropriate kinases in macrophages, neutrophils, and Kupffer cells (Supplemental Figure 5, A and B).

Compared with control Lyzs-cre transgenic mice, $\mathrm{p} 38 \delta^{\text {Lyz-KO }}$ and $\mathrm{p} 38 \gamma / \delta^{\mathrm{Lyz}-\mathrm{KO}}$ mice showed a delay in mortality in response to injection of D-gal+LPS (Figure 5A). These mice were also protected against liver damage and apoptosis, showing milder liver hemorrhaging, reduced serum levels of ALT and AST, and reduced caspase 3 cleavage (Figure 5, B-E). Analysis of cytokines revealed significantly lower circulating TNF- $\alpha$ and IL-6 in LPS-injected $\mathrm{p} 38 \delta^{\text {Lyz-KO }}$ and $\mathrm{p} 38 \gamma / \delta^{\text {Lyz-KO }}$ mice than in similarly treated Lyzs-cre controls (Figure 5F). Other chemokines involved in leukocyte migration (MIP1 $\alpha$ and MIP1 $\beta$ ) were also reduced (Supplemental Figure 6). In contrast, p38 $\gamma^{\text {Lyz-KO }}$ mice presented only a modest effect on circulating TNF- $\alpha$ levels and no differences in the levels of other cytokines (Figure 5F and Supplemental Figure 6).

The phenotype found in $\mathrm{p} 38 \gamma / \delta^{\text {Lyz-KO }}$ mice might reflect the function of these kinases in several myeloid cell populations implicated in D-gal+LPS damage (such as neutrophils, Kupffer cells, and macrophages). Analysis of these cell types in Lyzs-cre mice revealed expression of $\mathrm{p} 38 \gamma$ and $\mathrm{p} 38 \delta$ in macrophages, predominant expression of $\mathrm{p} 38 \delta$ in neutrophils, and exclusive expression of p38 $\gamma$ in Kupffer cells (Supplemental Figure 5A). Moreover, p38 $\gamma$ is strongly activated in Kupffer cells by LPS or TNF- $\alpha$ treatment (Supplemental Figure 5B), as judged by the impaired p38 phosphorylation observed by FACS in Kupffer cells from p38 $\gamma / \delta^{\text {Lyz-KO }}$ mice. Furthermore, analysis of phospho-p38 by Western blot confirmed that $\mathrm{p} 38 \gamma$ was the isoform activated in these cells after LPS treatment (Supplemental Figure 5C). LPS and TNF- $\alpha$ also activated p38 $\gamma$ and p $38 \delta$ in macrophages (Figure 6), while in neutrophils, p38 $\alpha$ was the only isoform activated after either stimulus (Supplemental Figure 5, B and D). Since neutrophils, Kupffer cells, and macrophages express p38 $\gamma, \mathrm{p} 38 \delta$, or both isoforms, any of these cell populations could have a role in the protection observed in p38 $\gamma / \delta^{\text {Lyz-KO }}$ mice. We therefore analyzed the different leukocyte populations that infiltrated the liver after D-gal+LPS injection. Analysis of liver-infiltrating leukocytes showed lower numbers of neutrophils in LPS-injected p38 $\gamma / \delta^{\text {Lyz-KO }}$ mice than in similarly treated Lyzs-cre mice. However, this reduction was not significant in LPS-injected p38 $8^{\text {Lyz-KO }}$ mice (Figure $5 \mathrm{G}$ ). None of the myeloidspecific p38-deficient mice showed alterations in the percentages of infiltrated macrophages or monocytes. Collectively, these data show that $\mathrm{p} 38 \gamma / \delta^{\mathrm{Lyz}-\mathrm{KO}}$ mice are more strongly affected than either of the single conditional knockouts; however, $\mathrm{p} 38 \delta^{\mathrm{Lyz}-\mathrm{KO}}$ animals have a stronger phenotype than $\mathrm{p} 38 \gamma^{\mathrm{Lyz}-\mathrm{KO}}$ mice, indicating partial 

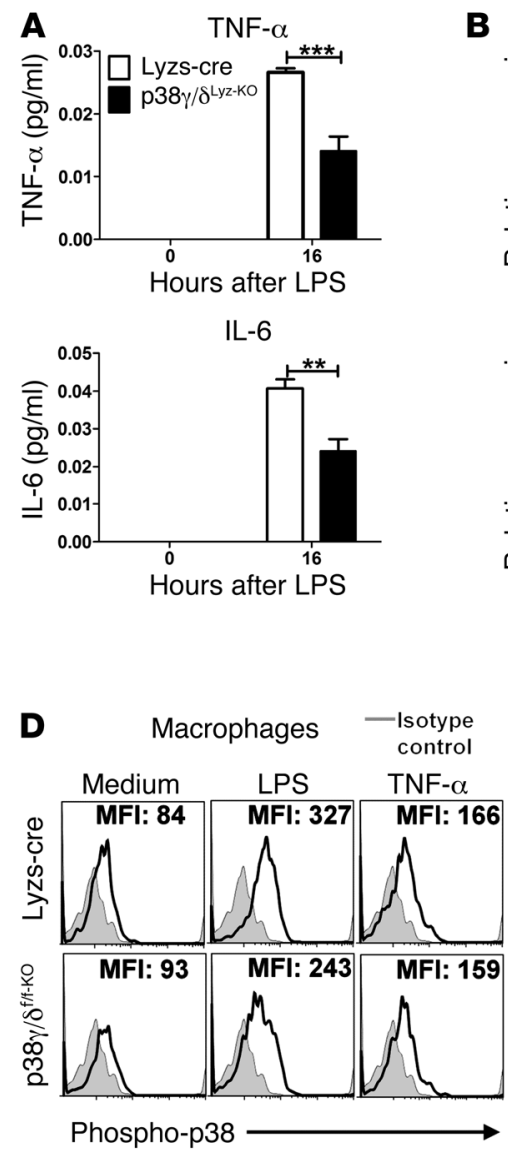

Phospho-p38

B

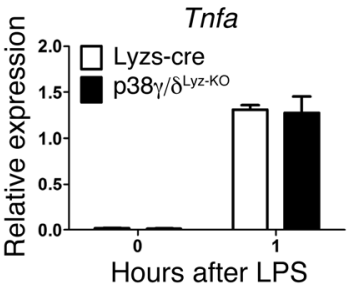

116

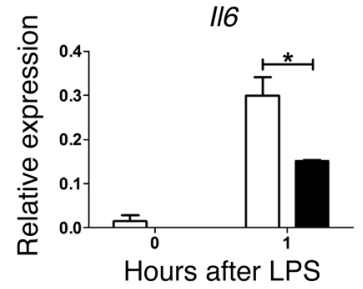

Phospho-p38

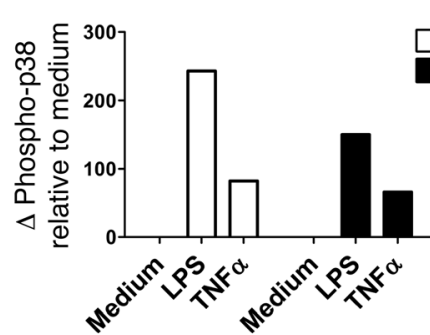

C

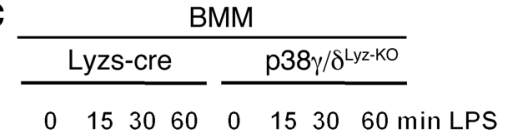

$\begin{array}{llllllll}0 & 15 & 30 & 60 & 0 & 15 & 30 & 60 \\ \text { min LPS }\end{array}$
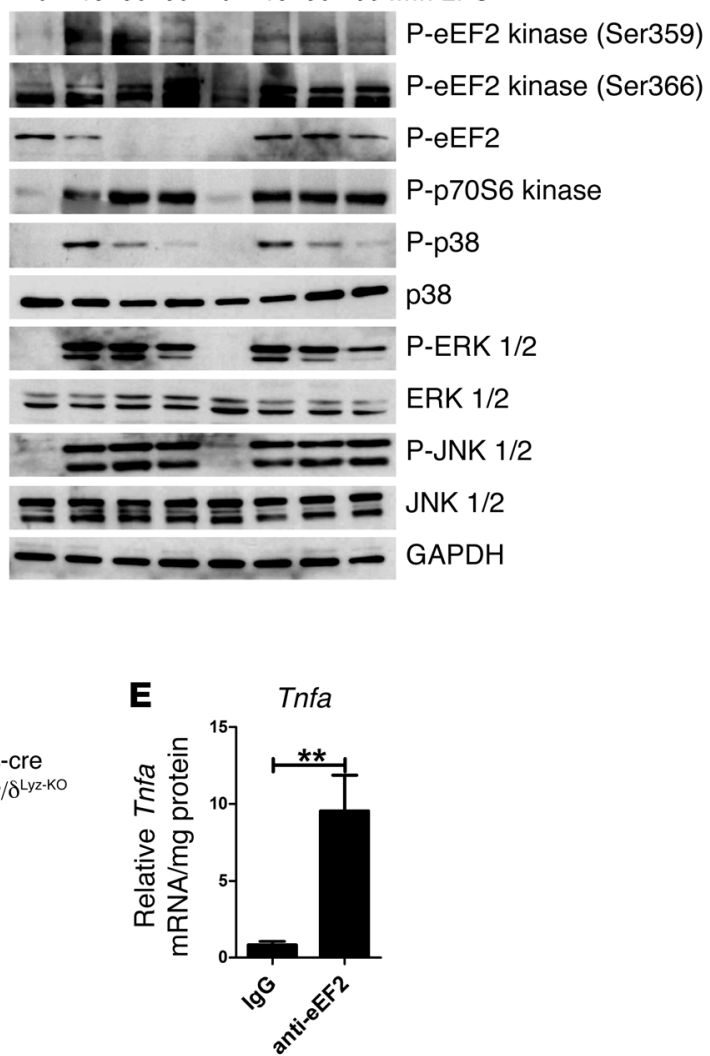

60 min after LPS

\section{F

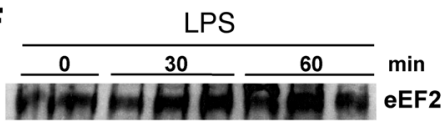

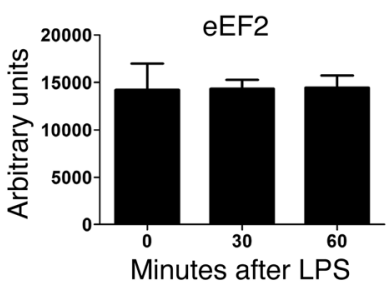
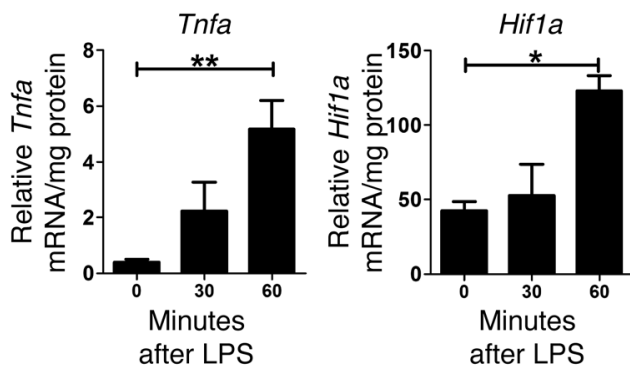

116

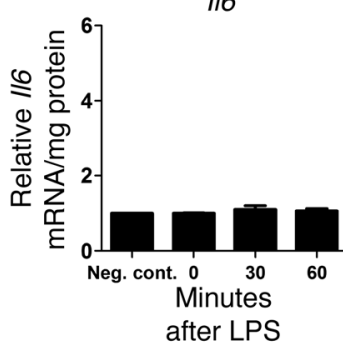

G

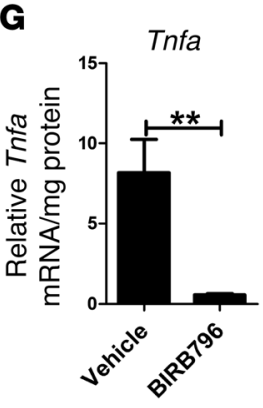

60 min after LPS

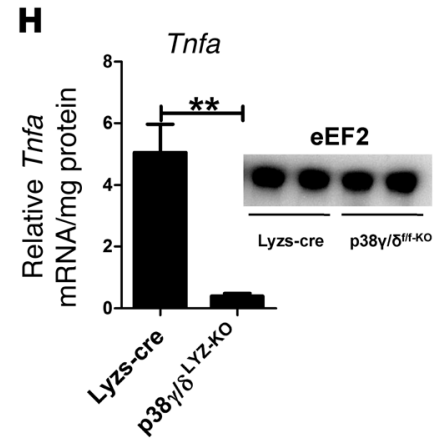

60 min after LPS

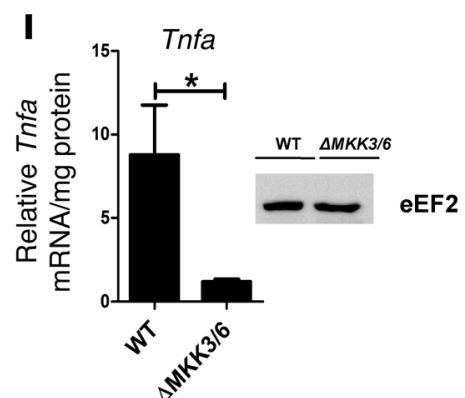

60 min after LPS 


\section{Figure 6}

p38 $\gamma / \delta^{\text {Lyz-KO }}$ macrophages promote TNF- $\alpha$ translation through phosphorylation of eEF2 kinase. (A-D) BM-derived macrophages from $\mathrm{p} 38 \gamma / \delta^{L y z}-\mathrm{KO}$ and Lyzs-cre mice were treated with LPS $(10 \mu \mathrm{g} / \mathrm{ml})$ or TNF- $\alpha(20 \mathrm{ng} / \mathrm{ml})$. Data were analyzed by 2-way ANOVA coupled to Bonferroni's post tests. (A) ELISA of TNF- $\alpha$ and IL-6 in supernatants. (B) qRT-PCR of Tnfa and II6 mRNA normalized to Gapdh. (C) Immunoblot of BM-derived macrophage (BMM) lysates. (D) Flow cytometry of phospho-p38 in BM-derived macrophages stimulated with LPS and TNF- $\alpha$ for 30 minutes. Intracellular phospho-p38 is depicted in black; gray corresponds to the isotype control. (E-I) Cells were treated with LPS. Tnfa mRNA was detected by qRT-PCR in immunoprecipitates (1 mg protein) obtained with anti-eEF2. mRNA amounts are expressed relative to the amount detected in control IgG immunoprecipitates. Data were analyzed by Student's $t$ test unless otherwise indicated. (E) Tnfa mRNA in RAW 264.7 cell immunoprecipitates. (F) Tnfa, Hif1a, and I/6 mRNA in RAW 264.7 cell immunoprecipitates. The presence of eEF2 was determined by immunoblot (upper panel) and quantified with ImageJ (1-way ANOVA coupled to Bonferroni's post tests). (G) RAW 264.7 cells were pretreated with DMSO or BIRB796 $(10 \mu \mathrm{M})$ for 30 minutes before stimulation with LPS (60 minutes). Tnfa mRNA was measured in immunoprecipitates. ( $\mathbf{H}$ and $\mathbf{I})$ Tnfa mRNA in immunoprecipitates of BM-derived macrophages from Lyzs-cre and $38 \gamma / \delta^{\text {Lyz-KO }}$ mice $(\mathrm{H})$ or WT or $\triangle \mathrm{MKK} 3 / 6$ mice (I) after stimulation (60 minutes). eEF2 in immunoprecipitates was detected by immunoblot (upper panel). Data are means $\pm \mathrm{SD}(n=4) .{ }^{*} P<0.05 ;{ }^{* \star} P<0.01$; ${ }^{\star \star \star} P<0.001$.

redundancy between $\mathrm{p} 38 \gamma$ and $\mathrm{p} 38 \delta$, with a predominant role of p38d. This overlapping action has been shown before and might account for the limited protection observed in single conditional knockouts $(36,37)$. We therefore used $\mathrm{p} 38 \gamma / \delta^{\mathrm{Lyz}-\mathrm{KO}}$ mice for further experiments. Further studies showed that the reduced neutrophil infiltration in liver is, in part, caused by lowered neutrophil mobilization from the BM (Figure $5 \mathrm{G}$ ), probably due to the defective production of TNF- $\alpha$. Furthermore, measurement of TNF- $\alpha-$ stimulated chemotaxis in a chamber assay revealed a slightly lowerthan-control mobility in p $38 \gamma / \delta^{\text {Lyz-KO }}$ neutrophils (Supplemental Figure 8A), and this effect was not increased by stimulation with a cytokine cocktail (Supplemental Figure 8B). Neutrophils produce several cytokines, and this function can be controlled by p $38 \alpha$ (29). Examination of cytokine production in our system showed that p $38 \gamma / \delta^{\text {Lyz-KO }}$ neutrophils express slightly reduced levels of TNF- $\alpha$ and IL- 6 after LPS treatment, but not sufficient to explain the phenotype observed (Supplemental Figure 8C).

To investigate whether the lower neutrophil infiltration in the livers of $\mathrm{p} 38 \gamma / \delta^{\text {Lyz-KO }}$ mice could account for the protection observed, we investigated whether neutrophil-specific depletion in Lyzs-cre mice results in milder liver damage upon D-gal+LPS treatment, similar to the phenotype of $\mathrm{p} 38 \gamma / \delta^{\mathrm{Lyz}-\mathrm{KO}}$ mice. GR 1 is highly expressed on neutrophils, and anti-GR1 antibody treatment has been shown to specifically deplete neutrophils (38). Treatment of mice with anti-GR1 one day before D-gal+LPS injection completely depleted $\mathrm{Gr} 1^{+}$neutrophils (Supplemental Figure 8D). Neutrophil depletion did not affect liver damage in Lyzs-cre mice, and neither did it abolish the protection observed in p38 $\gamma / \delta^{\text {Lyz-KO}}$, as assessed by the levels of ALT and AST, cleaved caspase 3, and PARP, or liver damage observed in H\&E-stained sections (Supplemental Figure 8, E-G).

These data indicate that neutrophils do not play a dominant role in the protection against D-gal+LPS-stimulated hepatic damage observed in $\mathrm{p} 38 \gamma / \delta^{\mathrm{Lyz}-\mathrm{KO}}$ mice and therefore indicate that a different myeloid cell type underlies this phenotype. Available evidence sug- gests an involvement of cytokine and chemokine production by activated Kupffer cells and infiltrated macrophages in the pathogenesis of liver damage (39). We therefore examined cytokine production by macrophages and Kupffer cells upon LPS treatment. ELISA analysis demonstrated that LPS-induced production of TNF- $\alpha$ and IL- 6 was lower in $\mathrm{p} 38 \gamma / \mathrm{\delta}^{\mathrm{Lyz}-\mathrm{KO}}$ macrophages and Kupffer cells than in Lyzscre control cells (Figure 6A and Supplemental Figure 7C). Moreover, only the supernatant from macrophages of $\mathrm{p} 38 \gamma / \delta^{\mathrm{Lyz}-\mathrm{KO}}$ animals was unable to induce apoptosis in primary hepatocytes (Supplemental Figure 7A), and analysis of this supernatant indicated a defect in cytokine production (Figure 6A and Supplemental Figure 7B). Similarly, induction of apoptosis by supernatant from control Lyzscre macrophages was blocked by addition of anti-TNF- $\alpha$ antibody, resulting in a protection similar to that obtained with $\mathrm{p} 38 \gamma / \delta^{\mathrm{Lyz}-\mathrm{KO}}$ supernatant (Supplemental Figure 7C). This finding thus indicates that the low TNF- $\alpha$ levels in p $38 \gamma / \delta^{\text {Lyz-KO }}$ macrophages and Kupffer cells could account for the protection against D-gal+LPS-induced liver damage.

$p 38 \gamma$ and $p 38 \delta$ mediate TNF- $\alpha$ translation in myeloid cells by phosphorylating eEF2K. p38 MAPK can promote cytokine expression at the level of transcription, after transcription (by promoting mRNA stability), and translation $(27,40)$. Il6 mRNA expression was reduced in $\mathrm{p} 38 \gamma / \mathrm{\delta}^{\mathrm{Lyz}-\mathrm{KO}}$ macrophages, but levels of Tnfa mRNA were comparable to those in Lyzs-cre macrophages (Figure 6B). The stability of Tnfa mRNA also showed no difference between the 2 genotypes (Supplemental Figure 7F). The translational action of p38 has been linked to eEF2 kinase, the enzyme that inactivates eEF2. This kinase is inhibited by p38 $\delta$-mediated phosphorylation on Ser359 and also by ribosomal protein S6 kinase-mediated phosphorylation on Ser366 $(19,23)$. Since little is known about the regulation of eEF2K in macrophages, we investigated the effect of $\mathrm{p} 38 \gamma / \delta$ deletion on the phosphorylation of eEF2K and its downstream substrate eEF2. LPS stimulation of Lyzs-cre macrophages induced eEF2K phosphorylation on Ser359, but this was markedly impaired in $\mathrm{p} 38 \gamma / \delta^{\mathrm{Lyz}-\mathrm{KO}}$ macrophages (Figure $6 \mathrm{C}$ ). This observation was also corroborated in Kupffer cells (Supplemental Figure 7D). eEF2K phosphorylation in Lyzs-cre macrophages correlated with dephosphorylation of eEF2, implying activation of elongation. In contrast, $\mathrm{p} 38 \gamma / \delta^{\mathrm{Lyz}-\mathrm{KO}}$ macrophages sustained high levels of eEF2 phosphorylation after the stimulus, suggesting that elongation is abolished.

To assess whether eEF2 controls TNF- $\alpha$ elongation, we examined the binding of eEF2 to Tnfa mRNA in immunoprecipitates of mRNA-eEF2 complexes in RAW cells. RAW extracts were incubated with anti-eEF2 or an isotype control antibody, and the presence of Tnfa mRNA in the immunoprecipitates was examined by quantitative (q) real-time PCR. High relative levels of Tnfa mRNA were detected in the anti-eEF2 immunoprecipitate, and this association was increased by LPS treatment (Figure 6, E and F). High mRNA levels for Hif1a, a known eEF2 target, were also detected in the anti-eEF2 immunoprecipitate (Figure $6 \mathrm{~F}$ ). In contrast, $I l 6 \mathrm{mRNA}$ levels did not differ between the anti-eEF2 and isotype control immunoprecipitates (Figure 6F). Pretreatment of RAW cells with BIRB796 abolished the association of eEF2 with Tnfa mRNA (Figure 6G). Moreover, Tnfa mRNA was detected in anti-eEF2 immunoprecipitates from LPS-treated Lyzs-cre or WT BM-derived macrophages but not in immunoprecipitates from similarly treated $\mathrm{p} 38 \gamma / \delta^{\mathrm{Lyz}-\mathrm{KO}}$ or $\Delta \mathrm{MKK} 3 / 6$ macrophages (Figure $6, \mathrm{H}$ and I). Together, our data indicate that Tnfa mRNA is a selective target of eEF2 and that 


\section{A}
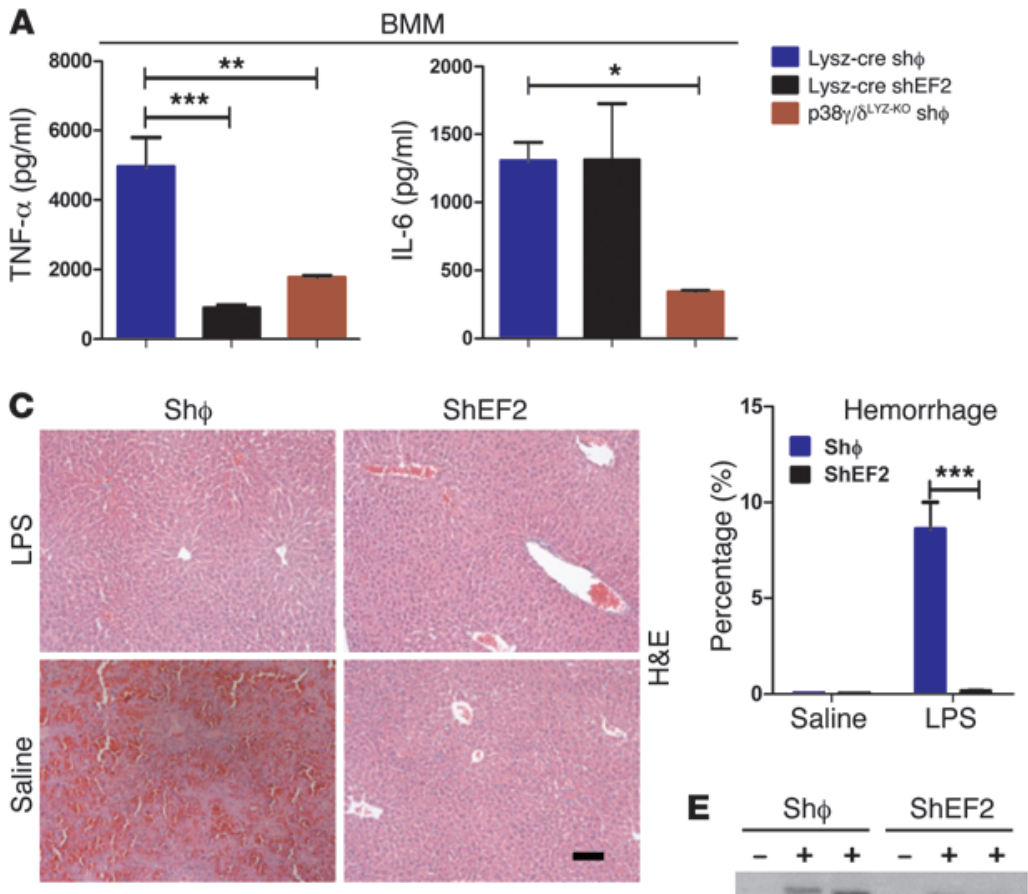

E

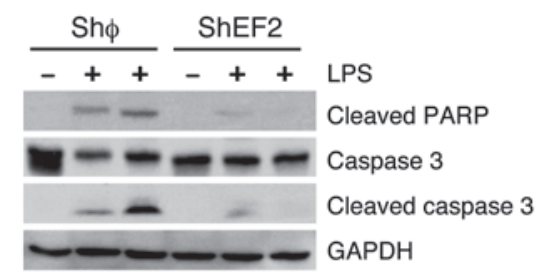

G

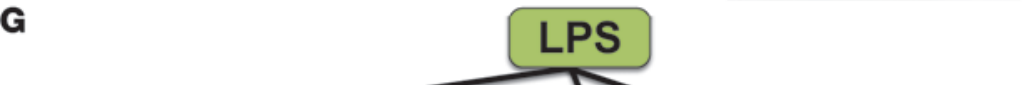

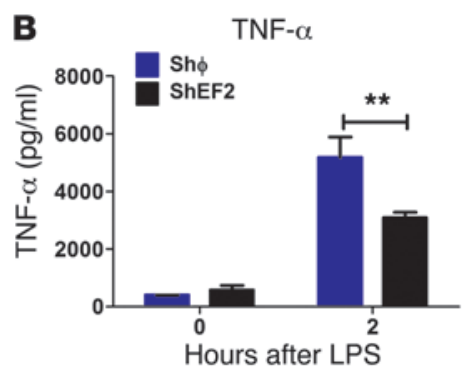

D

ALT
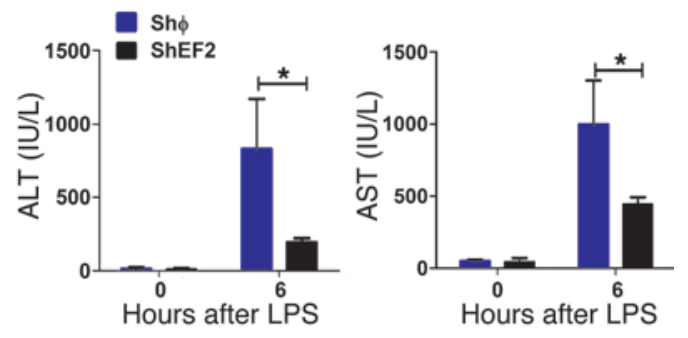

F

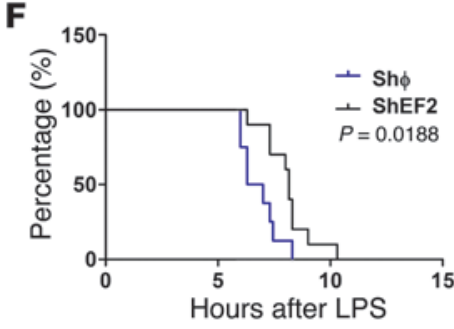

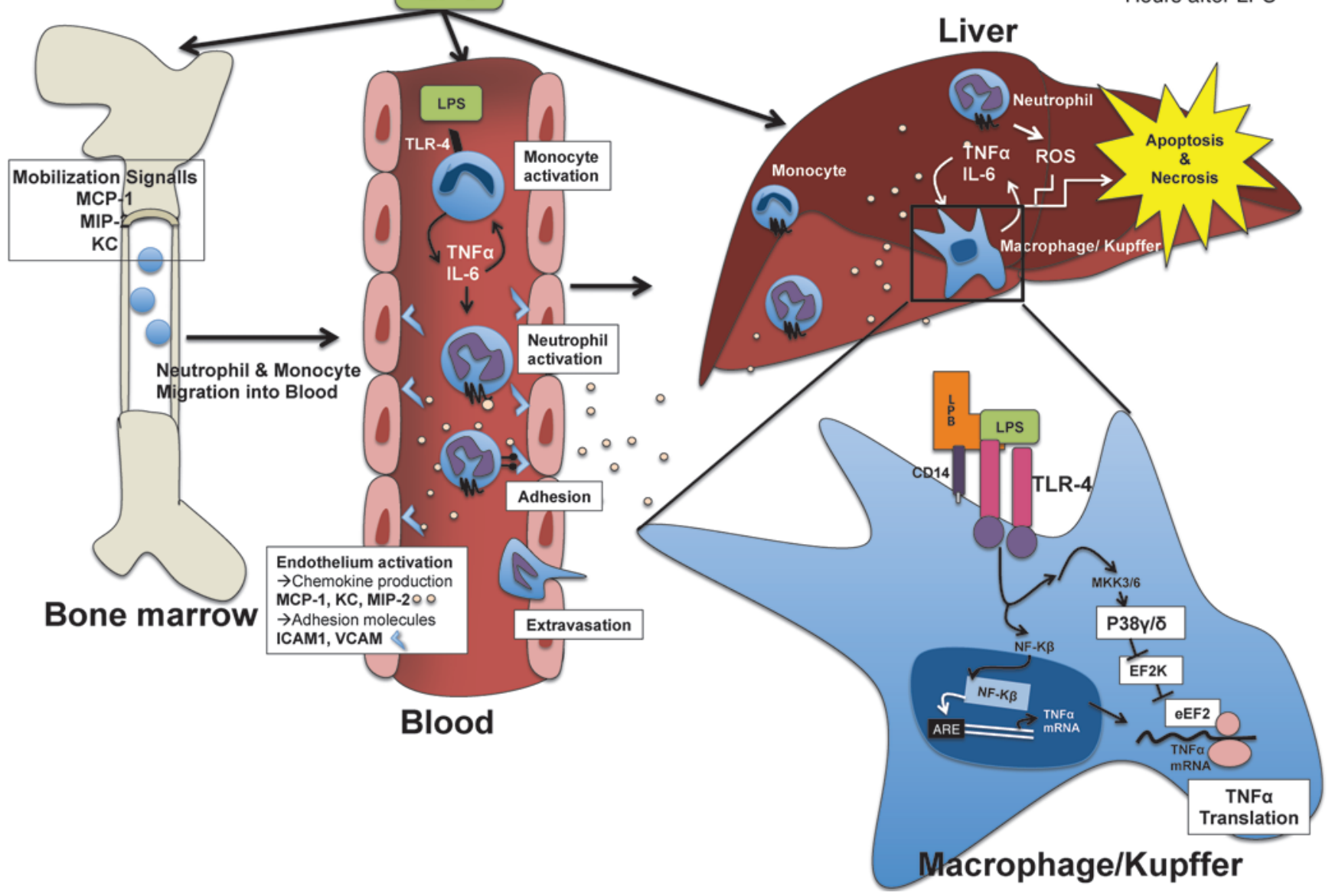




\section{Figure 7}

eEF2 controls TNF- $\alpha$ elongation, and its gene silencing protects against LPS-induced liver damage. (A) BM-derived macrophages from Lyzs-cre and $p 38 \gamma / \delta^{\text {Lyz-KO }}$ mice were transduced with GFP+ lentivirus expressing eEf2 shRNA (shEF2) or with empty vector (sh $\Phi$ ). ELISA of TNF- $\alpha$ and IL- 6 in culture supernatants was performed 16 hours after LPS $(10 \mu \mathrm{g} / \mathrm{ml})$ stimulation. One-way ANOVA coupled to Bonferroni's post tests $(n=6)$. (B-F) WT mice were i.v. injected with shEF2 or sh $\Phi$ $\mathrm{GFP}^{+}$lentiviral vectors. After 7 days, mice were injected with $\mathrm{D}$-gal+LPS or saline. Data are means $\pm \mathrm{SD}$. ${ }^{\star} P<0.05 ;{ }^{* \star} P<0.01 ;{ }^{* \star \star} P<0.001$ (2-way ANOVA coupled to Bonferroni's post tests). (B) ELISA of TNF- $\alpha$ and IL-6 in mouse serum 6 hours after treatment $(n=6)$. (C) Representative livers and H\&E-stained sections after removal at 6 hours after injection The chart presents hemorrhagic area as a percentage of the total area $(n=6)$. Scale bar: $50 \mu \mathrm{m}$. (D) Serum transaminase activity at 6 hours after injection $(n=6)$. (E) Immunoblot analysis of liver extracts $(n=6)$. (F) Mouse survival after D-gal+LPS injection $(n=10)$. Survival curves were created with the Kaplan-Meier method and compared by log-rank (Mantel-Cox) test. (G) The MKK3/6-p38 $/ / \delta$-eEF2K pathway is necessary for LPS-induced hepatitis. LPS induces leukocyte egress from BM and cytokine/chemokine production in peripheral blood and by Kupffer cells. This leads to massive leukocyte infiltration in the liver and hepatocellular injury. TNF- $\alpha$ plays a dominant role in LPS-induced liver injury. Activation of the MKK3/6-p38 $\gamma / \delta$-eEF2K pathway in macrophages/Kupffer cells controls TNF- $\alpha$ production at the mRNA elongation level in acute inflammatory responses to LPS.

the MKK3/6-p38 $\gamma / \delta$ pathway, by releasing eEF2K-mediated inhibition of eEF2 activity, promotes the translational elongation of TNF- $\alpha$ in macrophages.

eEF2 controls TNF- $\alpha$ translation elongation. To confirm eEF 2 control of TNF- $\alpha$ translation elongation, we used a recombinant lentivirus expressing an eEF2 shRNA (shEF2), which causes robust eEF2 knockdown in RAW cells (Supplemental Figure 9A). In macrophages, shEF2-mediated loss of eEF2 caused a decrease in TNF- $\alpha$ production of a magnitude similar to that caused by loss of $\mathrm{p} 38 \gamma / \delta$ (Figure 7A). In contrast, p38 $\gamma / \delta$-dependent IL-6 production was eEF2 independent (Figure 7A). These data indicate that the expression of TNF- $\alpha$ is controlled by eEF2, while the reduced IL-6 production in $\mathrm{p} 38 \gamma / \delta^{\mathrm{Lyz}-\mathrm{KO}}$ mice is caused by a different mechanism, most likely the loss of $\mathrm{p} 38 \gamma / \delta$-regulated $I l 6$ gene transcription.

To test the role of eEF2 in vivo, we injected mice with the shEF2 lentivirus or control shRNA lentivirus 1 week before exposure to D-gal+LPS. Blood levels of TNF- $\alpha$ were strongly reduced by the shEF2-injected mice compared with controls (Figure 7B). Moreover, this reduction in TNF- $\alpha$ was associated with protection against hepatic cell death, measured by cleavage of caspase 3 and PARP and protection against hepatic necrosis, and measured by liver hemorrhage and blood levels of ALT and AST (Figure 7, C-E). Collectively, these data indicate that $\mathrm{p} 38 \gamma / \delta$ MAPK proteins are required for the translation elongation of TNF- $\alpha$ in macrophages mediated by phosphorylation of eEF2K and the activation of eEF2.

\section{Discussion}

In this study,we show that protein kinases $\mathrm{p} 38 \gamma / \delta$ mediate the development of LPS-induced acute hepatitis by acting within a protein kinase signaling network that regulates the production of TNF- $\alpha$ by hematopoietic cells. MKK3 and MKK6 activate $\mathrm{p} 38 \gamma / \delta$ $(14,15)$, which in turn phosphorylate and inactivate eEF2 kinase (41). Once eEF2K is inactivated, eEF2 is dephosphorylated and activated, allowing the translational elongation of nascent TNF- $\alpha$. To our knowledge, this is the first report that eEF2 or $\mathrm{p} 38 \gamma / \delta$ con- trol cytokine production in myeloid cells. Our findings raise 3 key areas of interest: the specific role of myeloid cells, the involvement of $\mathrm{p} 38 \gamma / \delta$ compared with that of the other kinases of the same family, and how acute inflammatory responses can be controlled through tight regulation of translational elongation.

Myeloid cells such as Kupffer cells and other macrophages are well known to be a critical source of TNF- $\alpha$ in LPS-induced hepatitis $(14,32)$. This specific role of hematopoietic cells is consistent with our finding that the protective effects of MKK3/ 6 deficiency are also present in irradiated WT mice reconstituted with $\Delta \mathrm{MKK} 3 / 6 \mathrm{BM}$ (Figure 5). Furthermore, this protection was also observed in mice specifically lacking $\mathrm{p} 38 \gamma / \delta$ in the myeloid compartment (Figure 6). Two cell types of special relevance in acute hepatitis are neutrophils and monocytes/macrophages (including Kupffer cells), and both of these BM-derived cell populations are essential for the innate immune response (42). Monocytes/ macrophages bind to microbial constituents (such as LPS and cell wall constituents of Gram-positive bacteria), producing large amounts of pro- and antiinflammatory cytokines (3). Neutrophil recruitment and activation occur through TNF- $\alpha$-mediated chemokine production (3). This is evidenced by our finding that low expression of TNF- $\alpha$ in $\Delta$ MKK3/6 or $p 38 \gamma / \delta^{\text {Lyz-KO }}$ mice results in decreased chemokine production and neutrophil migration, an effect reversed by injection with TNF- $\alpha$. We did not find, however, any evidence for a role of $\mathrm{p} 38 \gamma / \delta$ in neutrophil migration, and we found only a minor effect on cytokine expression. This contrasts with the recent finding that $\mathrm{p} 38 \alpha$ can control cytokine production by neutrophils (29), illustrating the different roles and clinical potential of p38 kinase isoforms as targets for preventing LPS-induced damage.

Previous reports have identified p $38 \alpha$ as a key kinase involved in TNF- $\alpha$ production (43). p38 $\alpha$ MAPK deficiency in macrophages results in decreased TNF- $\alpha$ production, with a modest effect on IL-6 expression (44). However, specific inhibition of this kinase has been shown to be hepatotoxic, hindering its clinical use. For example, the p38 $\alpha$ inhibitor AMG 548 showed more than $85 \%$ inhibition of ex vivo LPS-induced TNF- $\alpha$ in healthy males, but its production and clinical use were suspended due to random liver enzyme elevations that were not dose or exposure dependent (45). Our results show that specific inhibition of $\mathrm{p} 38 \alpha / \beta$ with SB203580 intensifies D-gal+LPS-induced liver damage, whereas BIRB796, which inhibits all 4 p38 isoforms $(\alpha / \beta / \gamma / \delta)$ improves liver condition, including a reduction in apoptosis and necrosis. The toxicity associated with SB203580 might be caused by inhibition of $\mathrm{p} 38 \alpha$ in hepatocytes, since mice with specific p38 $\alpha$ deficiency in hepatocytes have increased JNK activity and increased susceptibility to liver damage $(17,43)$. In clinical use, BIRB796 also produces some liver enzyme elevations (46), most likely because of inhibition of $\mathrm{p} 38$. Our findings indicate that the generation of inhibitors that specifically target $\mathrm{p} 38 \gamma / \delta$ kinases might avoid the adverse effects found with $\mathrm{p} 38 \alpha$ inhibitors.

Our results further show that $\mathrm{p} 38 \gamma / \delta$ control macrophage production of TNF- $\alpha$ by promoting protein elongation during translation by eEF2. Protein synthesis is tightly regulated at transcriptional and posttranscriptional levels. Owing to the relatively long life time of mRNA transcripts, transcriptional regulation is commonly involved in slow, long-term cell responses, whereas immediate cell responses require posttranscriptional regulation of mRNA stability or translation. Two physiological situations requiring rapid regulation of protein synthesis are starvation and inflam- 
matory conditions. In starvation conditions, mTOR stops protein synthesis at the elongation step - the step that consumes most metabolic energy - by inhibiting eEF2, thereby preventing cell energy depletion. Less is known about the mechanisms involved in the termination of the inflammatory response once it has achieved its goal. This process needs to be rapidly regulated in order to avoid more tissue damage. Our results suggest that control of protein elongation might be an important mechanism by which cells rapidly shut down production of proinflammatory proteins that could extend tissue damage in the body, such as TNF- $\alpha$ during an inflammatory response. This might be an important mechanism by which cells tightly regulate cytokine production induced by stress kinases activated during inflammation.

In summary, we conclude that $\mathrm{p} 38 \gamma / \delta$ in myeloid cells promote TNF- $\alpha$ production by activating its translation without changes in mRNA levels. This is achieved by phosphorylation of eEF2K, releasing the inhibitory action of this kinase on eEF2. This posttranscriptional regulation might be an important mechanism regulating cytokine secretion during the innate immune response and provides potential targets for the treatment of liver diseases.

\section{Methods}

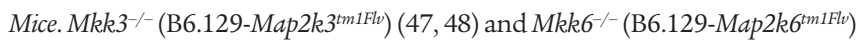

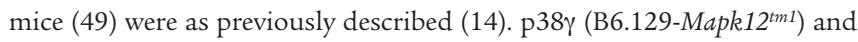
p388 (B6.129-Mapk13 ${ }^{\text {tm } 1}$ ) mice were crossed with B6.129P2-Lyz2tm1(cre)Ifo/J and backcrossed for 10 generations to the C57BL/6J background (Jackson Laboratory) and genotyped by PCR analysis of genomic DNA. Radiation chimeras were generated by exposing recipient mice to 2 doses of ionizing radiation $(525 \mathrm{~Gy})$ and reconstituting them with $2 \times 10^{7}$ donor BM cells by injection into the tail vein. Hepatitis was induced by i.p. injection with $50 \mu \mathrm{g} / \mathrm{kg}$ E. coli 0111:B LPS (Sigma-Aldrich) plus $1 \mathrm{~g} / \mathrm{kg}$ D-gal (Sigma-Aldrich) or by i.v. injection with $10 \mu \mathrm{g} / \mathrm{kg}$ TNF- $\alpha$ (R\&D Systems) plus $1 \mathrm{~g} / \mathrm{kg} \mathrm{GalN}$. When required, inhibitors were administered by i.p. injection at $15 \mathrm{mg} / \mathrm{kg}$ as previously reported $(50,51)$. For in vivo neutrophil depletion, $300 \mu \mathrm{g} /$ mouse of anti-Gr1 or the same volume of saline were i.v. injected 24 hours before challenge with LPS. Specificity of the depletion of cell populations was determined by flow cytometry of blood samples collected during the experiment.

Lentivirus vector production and infection of mice. Lentiviruses were produced as described (52). Transient calcium phosphate cotransfection of HEK293 cells was done with the pGIZP empty vector or PGIZP.shEF2 vector ( Thermo Scientific) together with $\mathrm{p} \Delta 8.9$ and pVSV-G. The supernatants containing the LV particles were collected 48 and 72 hours after removal of the calcium phosphate precipitate and were centrifuged at $700 \mathrm{~g}$ at $4{ }^{\circ} \mathrm{C}$ for 10 minutes and concentrated $(\times 165)$ by ultracentrifugation for 2 hours at $121986 \mathrm{~g}$ at $4^{\circ} \mathrm{C}$ (Ultraclear Tubes, SW28 rotor and Optima L-100 XP Ultracentrifuge; Beckman). Viruses were collected by adding cold sterile PBS and were titrated by qPCR.

Mice were injected in the tail vein with $200 \mu$ lentiviral particles suspended in PBS. Seven days after infection, mice were injected with LPS+D-gal.

Cell culture. Primary BM-derived macrophages were prepared and cultured as described previously (53). To isolate primary Kupffer cells from mouse livers, we performed collagenase perfusion and differential centrifugation using Percoll (Pharmacia) as described previously (54). BM neutrophils were purified by positive selection using biotinylated GR-1 (BD Biosciences - Pharmingen) and MACS streptavidin microbeads (Miltenyi Biotec). Neutrophil purity was $95 \%$ or greater, as assessed by flow cytometry (55). Cytokines in the culture medium were measured by multiplexed ELISA using a Luminex 200 analyzer (Millipore) and a mouse cytokine kit (Millipore). For primary hepatocyte isolation, liver parenchymal cells were prepared from 8- to 12-week-old anesthetized mice by in situ collagenase perfusion of the liver (56). Primary monolayer cultures were established by plating $1.5 \times 10^{5}$ viable cells $/ \mathrm{cm}^{2}$ in 6 -well collagen IV-coated plates. After 16 hours, primary hepatocytes were stimulated for 24 hours with TNF- $\alpha$ $(20 \mathrm{ng} / \mathrm{ml})$ plus CHX $(100 \mu \mathrm{g} / \mathrm{ml})$ or with $500 \mu \mathrm{l}$ of macrophage-conditioned medium for 12 hours. Neutrophil migration assays were performed using BD Falcon FluoroBlok 96 Multi-Well insert systems. Neutrophils were stained for 1 hour with $1.5 \mu \mathrm{M}$ calcein AM. Then, $1 \times 10^{5}$ cells were plated per well in the upper chamber in $50 \mu \mathrm{l}$ of culture medium without FBS. Lower chambers contained $200 \mu \mathrm{l}$ of culture medium alone or supplemented with the chemoattractant $(20 \mathrm{ng} / \mathrm{ml} \mathrm{TNF-} \alpha$ or $1 / 10$ cytokine cocktail). Fluorescence emission was measured at different time points with a fluorescent plate reader with bottom-reading capability (Fluoroskan Ascent; Thermo Scientific).

Serum analysis. Serum activities of ALT and AST were measured using the ALT and AST Reagent Kit (Biosystems Reagents) with a Benchmark Plus Microplate Spectrophotometer. Serum concentrations of cytokines were measured by multiplexed ELISA with a Luminex 200 analyzer (Millipore).

Biochemical analysis. Tissue extracts were prepared using Triton lysis buffer (20 mM Tris [pH 7.4], 1\% Triton X-100, 10\% glycerol, $137 \mathrm{mM} \mathrm{NaCl}$, $2 \mathrm{mM}$ EDTA, $25 \mathrm{mM} \beta$-glycerophosphate, $1 \mathrm{mM}$ sodium orthovanadate, $1 \mathrm{mM}$ phenylmethylsulfonyl fluoride, and $10 \mu \mathrm{g} / \mathrm{ml}$ aprotinin and leupeptin). Extracts ( $30 \mu \mathrm{g}$ protein) and immunoprecipitates (prepared from $2 \mathrm{mg}$ protein) were examined by protein immunoblot analysis with antibodies to ERK1/2, phospho-ERK1/2, phospho-MKK3/6, p38 $\alpha$ MAPK, phospho-p38, phospho-hsp27, phospho-JNK1/2, JNK1/2, caspase 3, cleaved caspase 3, cleaved PARP, and phospho-eEF2 kinase (Ser366), all from Cell Signaling and MKK3 (BD Biosciences - Pharmingen), MKK6 (Stressgen), and GAPDH and eEF2 (Santa Cruz Biotechnology Inc.). The anti-p38 $\gamma$ and $\mathrm{p} 38 \delta$ antibodies were raised against the peptides PPRQLGARVPKETAL and PIARKDSRRRSGMKL, respectively, which correspond to the $\mathrm{C}$ termini of the proteins.

Immunobistochemistry. Livers were fixed in $4 \%$ paraformaldehyde, processed, and embedded in paraffin. Sections $(5 \mu \mathrm{m})$ were stained with $\mathrm{H} \& \mathrm{E}$.

Isolation of liver-infiltrating mononuclear leukocytes. Mouse livers were collected, and a single-cell suspension was obtained and passed through a $70-\mu \mathrm{m}$ strainer. Leukocytes were collected from the interphase of centrifuged Ficoll gradients. Cells were counted with a CASY Cell Counter (57). Then $10^{6}$ Cells were labeled by surface staining (PE-conjugated anti-CD11b and PerCP-conjugated anti-Gr-1; Invitrogen), and another $10^{6}$ cells were stimulated in vitro with LPS $(10 \mu \mathrm{g} / \mathrm{ml})$ plus brefeldin A, which inhibits protein transport from ER to Golgi, leading to the accumulation of protein inside the ER (BD GolgiPlug) (1:1000) for 2 hours. The LPS-stimulated cells were then labeled by surface staining (PE-conjugated anti-CD11b and PerCP-conjugated anti-Gr-1; Invitrogen), fixed, permeabilized, and stained for intracellular TNF- $\alpha$ (APC-conjugated anti-TNF- $\alpha$ from BD Biosciences) and IL-12 (PE-conjugated anti-IL-12 from BD Biosciences). Flow cytometry was performed with a FACScan cytofluorometer (FACS Canto BD), and data were examined using FlowJo software.

Phospho-p38 FACS analysis. Primary myeloid cells were isolated (neutrophils, BM macrophages, and Kupffer cells) and stimulated for 30 minutes with LPS $(10 \mu \mathrm{g} / \mathrm{ml})$. Cells were fixed overnight in $70 \%$ methanol at $-20^{\circ} \mathrm{C}$ and stained with anti-phospho-p38 antibody (Cell Signaling) or control rabbit IgG followed by FITC-anti-rabbit IgG. Signal was detected by flow cytometry.

RNA-immunoprecipitation assay. RAW 264.7 cells (mouse leukemic monocyte macrophage cell line) were stimulated with LPS $(10 \mu \mathrm{g} / \mathrm{ml})$ and RNA-immunoprecipitation assays (RIPs) were performed using antibodies against eEF2 (Santa Cruz Biotechnology Inc.). The efficiency of immunoprecipitation was checked by immunoblot analysis. RNA was isolated from the input lysate, isotype control immunoprecipitate, and the anti-eEF2 immunoprecipitate. After reverse transcription, the levels of 
TNF- $\alpha$ mRNA were determined by qRT-PCR. Relative mRNA levels were calculated by subtracting background from the level of the target mRNA and then using the standard $\Delta \Delta \mathrm{C}_{\mathrm{T}}$ formula.

qRT-PCR. The expression of mRNA was examined by qRT-PCR using a 7900 Fast Real Time thermocycler and FAST SYBR GREEN assays (Applied Biosystems). Relative mRNA expression was normalized to Gapdh mRNA measured in each sample. Hif-1 $\alpha, G r-1, K C$ (Cxcl1), Mip-2 (Cxcl2), Mcp-1, and Icam-1 were amplified using the primers shown in Supplemental Table 1.

Statistics. Differences between groups were examined for statistical significance using 2-tailed Student's $t$ test or ANOVA coupled to Bonferroni's post-test. Kaplan-Meier analysis of survival was performed using the log-rank test.

Study approval. Animal studies were approved by the local ethics committee and by the IACUC of the University of Massachusetts Medical School. All animal procedures conformed to EU Directive 86/609/EEC and Recommendation 2007/526/EC regarding the protection of animals used for experimental and other scientific purposes, enacted under Spanish law $1201 / 2005$.

\section{Acknowledgments}

We thank J.M. Vazquez and E. Calvo for the proteomic analysis; T. Barrett and L.G. Leiva for technical assistance; S. Bartlett for English editing; and M. Rincón and F. Sanchez-Madrid for kindly revising and critically reading the article. Phospho-eEF2K (Ser359) antibody was provided by the Division of Signal Transduction
Therapy (DSTT), University of Dundee, Dundee, United Kingdom. p $\Delta 8.9$ and pVSV-G were kindly provided by Katia Urso and Juan Miguel Redondo (Centro Nacional de Investigaciones Cardiovasculares, Spain). FACS cytometry was conducted at the CNIC-Cellomics Unit. G. Sabio is an investigator of the Ramón y Cajal Program and a recipient of a Principe de Girona Impulsa award. R.J. Davis is an investigator of the Howard Hughes Medical Institute. Á. Verdugo is a recipient of a Madrid Regional Government fellowship. E. Manieri is a fellow of the La Caixa Foundation. Á. González-Rodríguez was supported by CIBERDEM (ISCIII, Spain). This work was funded by the following grants: ERC 260464, EFSD 2030, MICINN SAF2010-19347, and Comunidad de Madrid S2010/BMD-2326 (to G. Sabio:); MICINN SAF201127330 (to P. Martin); and MICINN SAF2012-3328 (to Á. Valverde). The CNIC is supported by the Ministry of Economy and Competitiveness and the Pro-CNIC Foundation.

Received for publication May 31, 2012, and accepted in revised form October 4, 2012.

Address correspondence to: Guadalupe Sabio, Vascular Biology and Inflammation, Centro Nacional de Investigaciones Cardiovasculares Carlos III, C/ Melchor Fernández Almagro, 3, 28029 Madrid, Spain. Phone: 34.91453.12.00, ext. 2004; Fax: 34.91.453.12.45; E-mail: gsabio@cnic.es.
1. Kim C, et al. The kinase p38 alpha serves cell typespecific inflammatory functions in skin injury and coordinates pro- and anti-inflammatory gene expression. Nat Immunol. 2008;9(9):1019-1027.

2. Wu Z, Han M, Chen T, Yan W, Ning Q. Acute liver failure: mechanisms of immune-mediated liver injury. Liver Int. 2010;30(6):782-794.

3. Antoniades CG, Berry PA, Wendon JA, Vergani D. The importance of immune dysfunction in determining outcome in acute liver failure. $J$ Hepatol. 2008;49(5):845-861.

4. Popa C, Netea MG, van Riel PL, van der Meer JW, Stalenhoef AF. The role of TNF-alpha in chronic inflammatory conditions, intermediary metabolism, and cardiovascular risk. J Lipid Res. 2007;48(4):751-762.

5. Wajant H, Pfizenmaier K, Scheurich P. Tumor necrosis factor signaling. Cell Death Differ. 2003;10(1):45-65.

6 . Bradley JR. TNF-mediated inflammatory disease. J Pathol. 2008;214(2):149-160.

7. Schwabe RF, Brenner DA. Mechanisms of Liver Injury. I. TNF-alpha-induced liver injury: role of IKK, JNK, and ROS pathways. Am J Physiol Gastrointest Liver Physiol. 2006;290:G583-G589.

8. Pfeffer KD, Huecksteadt TP, Hoidal JR. Expression and regulation of tumor necrosis factor in macrophages from cystic fibrosis patients. Am J Respir Cell Mol Biol. 1993;9(5):511-519.

9. Pasparakis M, Alexopoulou L, Douni E, Kollias G. Tumour necrosis factors in immune regulation: everything that's interesting is...new! Cytokine Growth Factor Rev. 1996;7(3):223-229.

10. Nebreda AR, Porras A. p38 MAP kinases: beyond the stress response. Trends Biochem Sci. 2000; 25(6):257-260

11. Schieven GL. The biology of p38 kinase: a central role in inflammation. Curr Top Med Chem. 2005;5(10):921-928.

12. Cuenda A, Rousseau S. p38 MAP-kinases pathway regulation, function and role in human diseases. Biochim Biophys Acta. 2007;1773(8):1358-1375.

13. Kyriakis JM, Avruch J. Mammalian mitogen-activated protein kinase signal transduction pathways activated by stress and inflammation. Physiol Rev. 2001;81(2):807-869.

14. Brancho D, et al. Mechanism of p38 MAP kinase activation in vivo. Genes Dev. 2003;17(16):1969-1978.

15. Remy G, et al. Differential activation of p38 MAPK isoforms by MKK6 and MKK3. Cell Signal. 2010;22(4):660-667.

16. Heinrichsdorff J, Luedde T, Perdiguero E, Nebreda AR, Pasparakis M. p38 alpha MAPK inhibits JNK activation and collaborates with IkappaB kinase 2 to prevent endotoxin-induced liver failure. $E M B O$ Rep. 2008;9(10):1048-1054.

17. Hui L, et al. p38alpha suppresses normal and cancer cell proliferation by antagonizing the JNK-c-Jun pathway. Nat Genet. 2007;39(6):741-749.

18. Beardmore VA, et al. Generation and characterization of p38beta (MAPK11) gene-targeted mice. Mol Cell Biol. 2005;25(23):10454-10464.

19. Knebel A, Morrice N, Cohen P. A novel method to identify protein kinase substrates: eEF2 kinase is phosphorylated and inhibited by SAPK4/p38delta. EMBO J. 2001;20(16):4360-4369.

20. Jung GA, et al. Methylation of eukaryotic elongation factor 2 induced by basic fibroblast growth factor via mitogen-activated protein kinase. Exp Mol Med. 2011;43(10):550-560.

21. Browne GJ, Proud CG. Regulation of peptide-chain elongation in mammalian cells. Eur J Biochem. 2002;269(22):5360-5368.

22. Wang L, Wang X, Proud CG. Activation of mRNA translation in rat cardiac myocytes by insulin involves multiple rapamycin-sensitive steps. $A m J$ Physiol Heart Circ Physiol. 2000;278(4):H1056-H1068.

23. Knebel A, Haydon CE, Morrice N, Cohen P. Stressinduced regulation of eukaryotic elongation factor 2 kinase by SB 203580 -sensitive and -insensitive pathways. Biochem J. 2002;367(pt 2):525-532.

24. Ono K, Han J. The p38 signal transduction pathway: activation and function. Cell Signal. 2000;12(1):1-13.

25. Mahtani KR, Brook M, Dean JL, Sully G, Saklatvala J, Clark AR. Mitogen-activated protein kinase p38 controls the expression and posttranslational modification of tristetraprolin, a regulator of tumor necrosis factor alpha mRNA stability. Mol Cell Biol. 2001;21(19):6461-6469.

26. Turpeinen T, Nieminen R, Moilanen E, Korhonen R. Mitogen-activated protein kinase phosphatase-1 negatively regulates the expression of interleukin-6, interleukin-8, and cyclooxygenase-2 in A549 human lung epithelial cells. J Pharmacol Exp Ther. 2010;333(1):310-318.

27. Nagaleekar VK, et al. Translational control of NKT cell cytokine production by 38 MAPK. J Immunol. 2011;186(7):4140-4146.

28. Kotlyarov A, et al. MAPKAP kinase 2 is essential for LPS-induced TNF-alpha biosynthesis. Nat Cell Biol. 1999;1(2):94-97.

29. Ramaiah SK, Jaeschke H. Role of neutrophils in the pathogenesis of acute inflammatory liver injury. Toxicol Pathol. 2007;35(6):757-766.

30. Alagbala Ajibade A, et al. TAK1 negatively regulates NF-kappaB and p38 MAP kinase activation in $\mathrm{Gr}-1(+) \mathrm{CD} 11 \mathrm{~b}(+)$ neutrophils. Immunity. 2012;36(1):43-54.

31. Xia S, Sha H, Yang L, Ji Y, Ostrand-Rosenberg S, Qi L. Gr-1+ CD11b+ myeloid-derived suppressor cells suppress inflammation and promote insulin sensitivity in obesity. J Biol Chem. 2011;286(26):23591-23599.

32. Dong Z, Wei H, Sun R, Tian Z. The roles of innate immune cells in liver injury and regeneration. Cell Mol Immunol. 2007;4(4):241-252.

33. Kuma Y, Sabio G, Bain J, Shpiro N, Marquez $\mathrm{R}$, Cuenda A. BIRB796 inhibits all p38 MAPK isoforms in vitro and in vivo. $J$ Biol Chem. 2005;280(20):19472-19479.

34. Cuenda A, et al. SB 203580 is a specific inhibitor of a MAP kinase homologue which is stimulated by cellular stresses and interleukin-1. FEBS Lett. 1995;364(2):229-233.

35. Clausen BE, Burkhardt C, Reith W, Renkawitz $\mathrm{R}$, Forster I. Conditional gene targeting in macrophages and granulocytes using LysMcre mice. Transgenic Res. 1999;8(4):265-277.

36. Sabio G, et al. Stress- and mitogen-induced phosphorylation of the synapse-associated protein SAP90/PSD-95 by activation of SAPK3/p38gamma and ERK1/ERK2. Biochem J. 2004;380(pt 1):19-30. 
37. Sabio G, et al. p38gamma regulates the localisation of SAP97 in the cytoskeleton by modulating its interaction with GKAP. EMBO J. 2005;24(6):1134-1145.

38. Jonsson F, et al. Mouse and human neutrophils induce anaphylaxis. JClin Invest. 2011;121(4):1484-1496.

39. Kolios G, Valatas V, Kouroumalis E. Role of Kupffer cells in the pathogenesis of liver disease. World J Gas troenterol. 2006;12(46):7413-7420.

40. Noubade R, et al. Activation of p38 MAPK in CD4 $\mathrm{T}$ cells controls IL-17 production and autoimmune encephalomyelitis. Blood. 2011;118(12):3290-3300.

41. Kotlyarov A, Gaestel M. Is MK2 (mitogen-activated protein kinase-activated protein kinase 2) the key for understanding post-transcriptional regulation of gene expression? Biochem Soc Trans. 2002;30(pt 6):959-963.

42. Shi $\mathrm{C}$, et al. Bone marrow mesenchymal stem and progenitor cells induce monocyte emigration in response to circulating toll-like receptor ligands. Immunity. 2011;34(4):590-601.

43. Lee JC, et al. A protein kinase involved in the regulation of inflammatory cytokine biosynthesis. Nature. 1994;372(6508):739-746.

44. Kang YJ, et al. Macrophage deletion of p38alpha partially impairs lipopolysaccharide-induced cellular activation. J Immunol. 2008;180(7):5075-5082.

45. Lee MR, Dominguez C. MAP kinase p38 inhibi- tors: clinical results and an intimate look at their interactions with p38alpha protein. Curr Med Chem. 2005;12(25):2979-2994

46. Schreiber S, et al. Oral p38 mitogen-activated protein kinase inhibition with BIRB 796 for active Crohn's disease: a randomized, double-blind, placebo-controlled trial. Clin Gastroenterol Hepatol. 2006;4(3):325-334

47. Wysk M, Yang DD, Lu HT, Flavell RA, Davis RJ. Requirement of mitogen-activated protein kinase kinase 3 (MKK3) for tumor necrosis factor-induced cytokine expression. Proc Natl Acad Sci U S A. 1999;96(7):3763-3768.

48. Lu HT, et al. Defective IL-12 production in mitogenactivated protein (MAP) kinase kinase 3 (Mkk3)deficient mice. EMBO J. 1999;18(7):1845-1857.

49. Tanaka N, et al. Differential involvement of p38 mitogen-activated protein kinase kinases MKK3 and MKK6 in T-cell apoptosis. EMBO Rep. 2002;3(8):785-791.

50. Chopra P, et al. Pharmacological profile of AW-814141, a novel, potent, selective and orally active inhibitor of p38 MAP kinase. Int Immunopharmacol. 2010;10(4):467-473.

51. Sukhtankar D, et al. Inhibition of p38-MAPK signaling pathway attenuates breast cancer induced bone pain and disease progression in a murine model of cancer-induced bone pain. Mol Pain. 2011;7:81

52. Urso K, et al. NFATc3 regulates the transcription of genes involved in T-cell activation and angiogenesis. Blood. 2011;118(3):795-803.

53. Kim L, Butcher BA, Denkers EY. Toxoplasma gondii interferes with lipopolysaccharide-induced mitogen-activated protein kinase activation by mechanisms distinct from endotoxin tolerance. Immunol. 2004;172(5):3003-3010.

54. Kuboki S, et al. Hepatocyte NF-kappaB activation is hepatoprotective during ischemia-reperfusion injury and is augmented by ischemic hypothermia. Am J Physiol Gastrointest Liver Physiol. 2007;292(1):G201-G207.

55. Partida-Sanchez S, et al. Chemotaxis of mouse bone marrow neutrophils and dendritic cells is controlled by adp-ribose, the major product generated by the CD38 enzyme reaction. J Immunol. 2007;179(11):7827-7839.

56. Mayoral R, Valverde AM, Llorente Izquierdo C, Gonzalez-Rodriguez A, Bosca L, Martin-Sanz P. Impairment of transforming growth factor beta signaling in caveolin-1-deficient hepatocytes: role in liver regeneration. J Biol Chem. 2010;285(6):3633-3642.

57. Cruz-Adalia A, et al. CD69 limits the severity of cardiomyopathy after autoimmune myocarditis. Circulation. 2010;122(14):1396-1404 